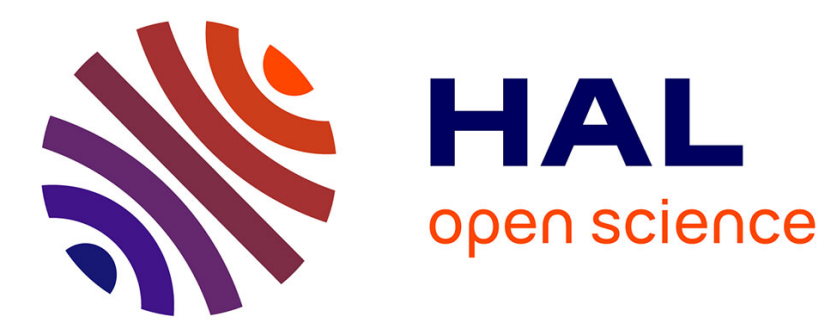

\title{
Piecewise polynomial chaos expansion with an application to brake squeal of a linear brake system
}

Emmanuelle Sarrouy, Olivier Dessombz, Jean-Jacques Sinou

\section{To cite this version:}

Emmanuelle Sarrouy, Olivier Dessombz, Jean-Jacques Sinou. Piecewise polynomial chaos expansion with an application to brake squeal of a linear brake system. Journal of Sound and Vibration, 2013, 332, pp.577-594. 10.1016/j.jsv.2012.09.009 . hal-00744841

\section{HAL Id: hal-00744841 https://hal.science/hal-00744841}

Submitted on 24 Oct 2012

HAL is a multi-disciplinary open access archive for the deposit and dissemination of scientific research documents, whether they are published or not. The documents may come from teaching and research institutions in France or abroad, or from public or private research centers.
L'archive ouverte pluridisciplinaire HAL, est destinée au dépôt et à la diffusion de documents scientifiques de niveau recherche, publiés ou non, émanant des établissements d'enseignement et de recherche français ou étrangers, des laboratoires publics ou privés. 


\title{
Piecewise polynomial chaos expansion with an application to brake squeal of a linear brake system
}

\author{
E. Sarrouy, ${ }^{\mathrm{a}, *}$ O. Dessombz ${ }^{\mathrm{a}}$ JJ. Sinou ${ }^{\mathrm{a}}$ \\ ${ }^{a}$ Ecole Centrale de Lyon, Laboratoire de Tribologie et Dynamique des Systèmes (UMR CNRS 5513), 36 avenue Guy de Collongue, 69134 Ecully Cedex, \\ France
}

\begin{abstract}
This paper proposes numerical developments based on polynomial chaos (PC) expansions to process stochastic eigenvalue problems efficiently. These developments are applied to the problem of linear stability calculations for a simplified brake system: the stability of a finite element model of a brake is investigated when its friction coefficient or the contact stiffness are modeled as random parameters. Getting rid of the statistical point of view of the PC method but keeping the principle of a polynomial decomposition of eigenvalues and eigenvectors, the stochastic space is decomposed into several elements to realize a low degree piecewise polynomial approximation of these quantities. An approach relying on continuation principles is compared to the classical dichotomy method to build the partition. Moreover, a criterion for testing accuracy of the decomposition over each cell of the partition without requiring evaluation of exact eigenmodes is proposed and implemented.

Several random distributions are tested, including a uniform-like law for description of friction coefficient variation. Results are compared to Monte Carlo simulations so as to determine the method accuracy and efficiency. Some general rules relative to the influence of the friction coefficient or the contact stiffness are also inferred from these calculations.
\end{abstract}

Keywords: brake squeal noise, stochastic, friction, eigenvalue problem

\section{Introduction}

Brake squeal noise is a real problem for the automotive [1,2] and railway [3] industry: it is a discomfort for passengers, generates high warranty costs for car manufacturers and causes problems to train operators that have to face claims of people living next to train stations. Indeed, this complex phenomenon occurs at low speeds. It is acknowledged to come from the vibration of the brake components with high and audible frequencies and high intensity (up to $130 \mathrm{~dB}$ ).

Many deterministic studies are available in the literature using different mechanisms to explain brake squeal and going from the simplest models with a few degrees of freedom only [4] to large finite element models taking many components into account [5]. Among the mechanisms put forward to explain this noise generation, one finds the decreasing friction coefficient with sliding speed, sprag-slip [6], stick-slip [7, 8] and self-excited vibration coming from mode coupling with a constant friction coefficient $[9,10]$. Besides the above mentioned references, an extensive review is available in [11, 12].

In this paper, a simple Coulomb friction law with a constant friction coefficient is used. Instability then occurs when two modes couple. This can be detected by analyzing the eigenvalues of the linear problem or the tangent one if non-linear effects are considered. In the latter case it is also possible to determine the limit cycles using frequency domain methods [13, 14] or to conduct a transient analysis $[15,16]$. In this work, we focus our attention on the stability analysis of a linear brake system with uncertainties. The stability of the equilibrium of the linear system is investigated via the Complex Eigenvalue Analysis (CEA) method. The eigenvalue problem has then to be solved. The objective of the present study is to propose a methodology and numerical developments based on polynomial chaos expansions (PCE) to process stochastic eigenvalue problems accurately and efficiently and to save time compared to Monte Carlo simulations. As no non-linear phenomena are considered, the prediction of squeal frequencies are limited. Nonetheless, it is a first step towards faster calculations for processing brake systems including uncertainties.

The present model is a linear finite element model (FEM) whose equilibrium stability is investigated through the corresponding eigenvalue problem. Instead of refining the model to get precise information on its vibratory behavior, some parameters are considered to be random. As pointed out by several papers $[17,18]$, some parameters such as the friction coefficient are hard to characterize accurately for each brake and each braking action. It then seems appropriate to conduct a stochastic study to take into account this variability.

The main problems encountered for a stochastic study is the description of the random inputs and the numerical cost [19]. Indeed, a well known and robust method is the Monte Carlo simulation (MCS). It consists in the evaluation of quantities of interest (for example here, the eigenvalues) for a large sample of uncertain inputs values. Statistical estimators (such as the mean, standard deviation, etc.) can then be rebuilt (see for example [20] for MCS applied to a brake model). For systems

\footnotetext{
*Corresponding author. Tel: +33 472186464/Fax:+33 472189144

Email addresses: emmanuelle.sarrouy@(ec-lyon.fr,gmail.com) (E. Sarrouy), olivier.dessombz@ec-lyon.fr (O. Dessombz), jean-jacques.sinou@ec-lyon.fr (JJ. Sinou)
} 
with more than a few degrees of freedom, this operation can have a prohibitive cost in terms of computation time even though recent developments relying in particular on parallel processing [19] let MCS remain an attractive method. Apart from MCS, three other classes of methods were developed [19]. First the perturbation methods are based on Taylor series expansion on a set of zero mean random variables [21,22]. The series are usually truncated to first or second order to avoid difficulties linked to the evaluation of higher order derivatives. Hence they are mostly used in the case of small variation of random inputs. It is to be noted that recent developments allow the computation of complex eigenvalues [23, 24]. The second family of methods relies on the so-called Polynomial Chaos Expansions and was first introduced in the field of structural dynamics by Ghanem and Spanos [25]. It basically consists in the decomposition of quantities of interest on an orthogonal basis of polynomials (see for example [26] for a detailed presentation of such methods). The procedure proposed in this paper derives from PCE and its further developments. A new method to build the stochastic space partition is proposed, based on continuation method principles. Moreover, an efficient accuracy criterion dedicated to the eigenvalue problem is developed to test the current polynomial decomposition over each element of the partition without having to compare it to any exact result. These several improvements aim at providing a method that returns accurate results in a lesser time. Section 2 exposes the method in detail. Finally, Soize [27, 28] developed a non-parametric approach which does not require identification of the uncertain local parameters. A deeper overview of these several methods is available in successive review papers by Ibrahim et al. [29, 30] and Schüeller et al. [19, 31, 32].

This paper is organized as follows: first, Section 2 exposes the theoretical background. Then the stochastic study of a FEM brake model is performed using the proposed method for several random laws. Accuracy and efficiency of the method compared to MCS is evaluated and the stochastic results about system stability are commented.

\section{Theoretical background used to process uncertainty}

This section is devoted to the presentation of the mathematical background of the method proposed in order to handle the eigenvalue problem of uncertain systems. First, quick recalls are proposed about the stability study of equilibria through the tangent eigenvalue problem. Then, the general idea of using an approximation of quantities of interest by polynomial decomposition is studied. Finally, a refinement of this approach that enables the study of uncertain quantities with strong variations is presented. The last part is dedicated to the algorithm that organizes theses different points.

\subsection{Deterministic study of stability through the eigenvalue problem}

The deterministic equation for a general linear structure is:

$$
\mathbf{M} \ddot{\mathbf{q}}+\mathbf{D} \dot{\mathbf{q}}+\mathbf{K q}=\mathbf{f}_{\mathrm{e}}
$$

where $\mathbf{q}, \dot{\mathbf{q}}$ and $\ddot{\mathbf{q}}$ denote the vector of degrees of freedom (dofs) and its first and second derivatives with respect to time. $\mathbf{M}, \mathbf{D}$ and $\mathbf{K}$ are the mass, damping and stiffness matrices respectively and $\mathbf{f}_{\mathrm{e}}$ is the vector of excitation forces. The static equilibrium for such a formulation is solution of $\mathbf{K q} \mathbf{q}_{s}=\mathbf{f}_{\mathrm{e}}$. Its stability depends on real parts of eigenvalues of the following problem [33]:

$$
\mathbf{A} \mathbf{u}_{k}=\lambda_{k} \mathbf{B} \mathbf{u}_{k}, \text { with } \mathbf{A}=\left[\begin{array}{cc}
\mathbf{0} & \mathbf{K} \\
-\mathbf{K} & -\mathbf{D}
\end{array}\right] \text { and } \mathbf{B}=\left[\begin{array}{cc}
\mathbf{K} & \mathbf{0} \\
\mathbf{0} & \mathbf{M}
\end{array}\right]
$$

Computing the $2 n$ eigenvalues $\lambda_{k}$ and the matching eigenvectors $\mathbf{u}_{k}$ solutions of Eq. (2) (where $n$ denotes the system size), one can determine the stability of the equilibrium: if there exists at least one eigenvalue with strictly positive real part, then it is unstable, otherwise, this is a stable equilibrium position. Moreover, in order to define the eigenvectors $\mathbf{u}_{k}$ uniquely, one will use normalization with respect to $\mathbf{B}$ matrix:

$$
\mathbf{u}_{k}^{\mathrm{T}} \mathbf{B} \mathbf{u}_{k}=1
$$

\subsection{Introducing uncertainties}

If some parts of the structure have uncertain properties, they can be rendered by adding uncertain terms to the dynamic system (1):

$$
(\mathbf{M}+\tilde{\mathbf{M}}) \ddot{\mathbf{q}}+(\mathbf{D}+\tilde{\mathbf{D}}) \dot{\mathbf{q}}+(\mathbf{K}+\tilde{\mathbf{K}}) \mathbf{q}=\mathbf{f}_{\mathrm{e}}
$$

Random contributions are denoted with tilde notation. It is assumed that they have zero mean and depend on a set of random parameters denoted $\boldsymbol{\xi}$.

The stability study of the static equilibria of Eq. (4) is then achieved through the following eigenvalue problem

$$
(\mathbf{A}+\tilde{\mathbf{A}}) \tilde{\mathbf{u}}_{k}=\tilde{\lambda}_{k}(\mathbf{B}+\tilde{\mathbf{B}}) \tilde{\mathbf{u}}_{k}
$$

with normalization equation

$$
\tilde{\mathbf{u}}_{k}^{\mathrm{T}}(\mathbf{B}+\tilde{\mathbf{B}}) \tilde{\mathbf{u}}_{k}=1
$$

where $\tilde{\mathbf{u}}_{k}$ and $\tilde{\lambda}_{k}$ denote the $k$-th stochastic eigenvector and eigenvalue respectively. 
The proposed method to compute these stochastic eigenmodes uses a decomposition of uncertain quantities on a polynomial basis. Depending on the law followed by the set of random parameters $\boldsymbol{\xi}$, some basis are optimal considering the convergence rate with polynomial degree [34]. For example Hermite polynomials are most adapted to normal law while Legendre polynomials suit best the uniform law. One of the most popular methods based on such a polynomial decomposition is the Polynomial Chaos expansion which uses a basis of Hermite multivariate polynomials [25, 35]. A previous work by the authors addresses the dispersion of a finite element rotor modes using such a basis [36]. Recently, this method has been extended to the polynomials of the Askey scheme leading to the so-called "generalized Polynomial Chaos" [37]. Note that the expression "polynomial chaos" refers to the theory following Wiener's developments [38] and is to be distinguished from the "deterministic chaos" [39].

The rest of this section is dedicated to the presentation of the method adapted to the stochastic normalized eigenvalue problem (5) and (6).

To this end, let us denote $\boldsymbol{\psi}_{n}, 1 \leq n \leq N$ the orthogonal polynomial basis with $N$ elements used for uncertain quantities decomposition and $<\boldsymbol{\psi}_{i}, \boldsymbol{\psi}_{j}>$ the associated scalar product.

First, matrices introducing uncertainty are decomposed as follows, using a Karhunen-Loève decomposition or assuming them so regarding experimental results [25, 37]:

$$
\tilde{\mathbf{X}}=\sum_{n=2}^{N_{X}} \mathbf{X}_{n} \boldsymbol{\psi}_{n}(\boldsymbol{\xi})
$$

where $\mathbf{X}$ can denote $\mathbf{M}, \mathbf{D}$ or $\mathbf{K} . \boldsymbol{\psi}_{1}$ will conventionally denote the constant polynomial equal to 1 (associated with deterministic component); this implies that polynomials with index greater or equal to 2 are zero mean.

Then stochastic eigenvalues and eigenvectors are decomposed on both the deterministic eigenmodes and the polynomial basis:

$$
\begin{gathered}
\tilde{\lambda}_{k}=\lambda_{k} \sum_{s=1}^{N}\left({ }^{(k)} a_{s}+\mathrm{j}^{(k)} b_{s}\right) \boldsymbol{\psi}_{s}(\boldsymbol{\xi}) \\
\tilde{\mathbf{u}}_{k}=\sum_{p \in \mathcal{P}_{k}}\left({ }^{(k)} \tilde{\gamma}_{p}(\boldsymbol{\xi})+\mathrm{j}^{(k)} \tilde{\mu}_{p}(\boldsymbol{\xi})\right) \mathbf{u}_{p}
\end{gathered}
$$

with

$$
{ }^{(k)} \tilde{\gamma}_{p}(\boldsymbol{\xi})=\sum_{n=1}^{N}{ }^{(k)} \gamma_{p}^{n} \boldsymbol{\psi}_{n}(\boldsymbol{\xi}) \text { and }{ }^{(k)} \tilde{\mu}_{p}(\boldsymbol{\xi})=\sum_{n=1}^{N}{ }^{(k)} \mu_{p}^{n} \boldsymbol{\psi}_{n}(\boldsymbol{\xi})
$$

giving

$$
\tilde{\mathbf{u}}_{k}=\sum_{p \in \mathcal{P}_{k}}\left(\sum_{n=1}^{N}\left({ }^{(k)} \gamma_{p}^{n}+\mathrm{j}^{(k)} \mu_{p}^{n}\right) \boldsymbol{\psi}_{n}(\boldsymbol{\xi})\right) \mathbf{u}_{p}
$$

where the coefficients ${ }^{(k)} \gamma_{p}^{n},{ }^{(k)} \mu_{p}^{n},{ }^{(k)} a_{s}$ and ${ }^{(k)} b_{s}$ are real and $\mathrm{j}$ denotes the imaginary unit $\left(\mathrm{j}^{2}=-1\right) . \mathcal{P}_{k}$ is the set of deterministic modes used for the $k$-th stochastic mode decomposition. It contains $P_{k}$ elements. This decomposition is a generalization of what is proposed in [40]. The use of complex weights ${ }^{(k)} \gamma_{p}^{n}+\mathrm{j}^{(k)} \mu_{p}^{n}$ and ${ }^{(k)} a_{s}+\mathrm{j}^{(k)} b_{s}$ lets real and imaginary parts of stochastic eigenvectors and eigenvalues evolve independently. The selection of a few deterministic modes included in $\mathcal{P}_{k}$ instead of using all the deterministic modes $\left(P_{k}=2 n\right)$ can lead to a great computation time reduction and is addressed in Section 3.3.

To get the final system of equations that leads to the unknowns ${ }^{(k)} \gamma_{p}^{n},{ }^{(k)} \mu_{p}^{n},{ }^{(k)} a_{s}$ and ${ }^{(k)} b_{s}$, equations (5) and (6) are first projected onto the polynomial basis $\psi_{n}, 1 \leq n \leq N$ using the dedicated scalar product. This gives the set of equations (12) and (13) for $1 \leq i \leq N$.

$$
\begin{aligned}
& \sum_{p \in \mathcal{P}_{k}} \sum_{n_{A}=1}^{N_{A}}\left(\mathbf{A}_{n_{A}} \mathbf{u}_{p}\right)\left(\sum_{n=1}^{N}\left({ }^{(k)} \gamma_{p}^{n}+\mathrm{j}^{(k)} \mu_{p}^{n}\right)<\boldsymbol{\psi}_{n_{A}} \boldsymbol{\psi}_{n}, \boldsymbol{\psi}_{i}>\right) \\
&-\lambda_{k} \sum_{p \in \mathcal{P}_{k}} \sum_{n_{B}=1}^{N_{B}}\left(\mathbf{B}_{n_{B}} \mathbf{u}_{p}\right) \times \\
&\left(\sum_{n, s=1}^{N}\left({ }^{(k)} \gamma_{p}^{n}+\mathrm{j}^{(k)} \mu_{p}^{n}\right)\left({ }^{(k)} a_{s}+\mathrm{j}^{(k)} b_{s}\right)<\boldsymbol{\psi}_{n_{B}} \boldsymbol{\psi}_{s} \boldsymbol{\psi}_{n}, \boldsymbol{\psi}_{i}>\right)=\mathbf{0}
\end{aligned}
$$

and

$$
\begin{aligned}
& \sum_{p_{1}, p_{2} \in \mathcal{P}_{k}} \sum_{n_{B}=1}^{N_{B}}\left(\mathbf{u}_{p_{1}}^{\mathrm{T}} \mathbf{B}_{n_{B}} \mathbf{u}_{p_{2}}\right) \times \\
& \left(\sum_{n_{1}, n_{2}=1}^{N}\left({ }^{(k)} \gamma_{p_{1}}^{n_{1}}+\mathrm{j}^{(k)} \mu_{p_{1}}^{n_{1}}\right)\left({ }^{(k)} \gamma_{p_{2}}^{n_{2}}+\mathrm{j}^{(k)} \mu_{p_{2}}^{n_{2}}\right)<\boldsymbol{\psi}_{n_{B}} \boldsymbol{\psi}_{n_{1}} \boldsymbol{\psi}_{n_{2}}, \boldsymbol{\psi}_{i}>\right) \\
& \quad-1<\boldsymbol{\psi}_{1}, \boldsymbol{\psi}_{i}>=0
\end{aligned}
$$

where $\mathbf{A}_{n_{A}}$ and $\mathbf{B}_{n_{B}}$ are the matrices used to decompose random matrices $\tilde{\mathbf{A}}$ and $\tilde{\mathbf{B}}$ respectively, in accordance with Eq. (7) notation. 
(a)

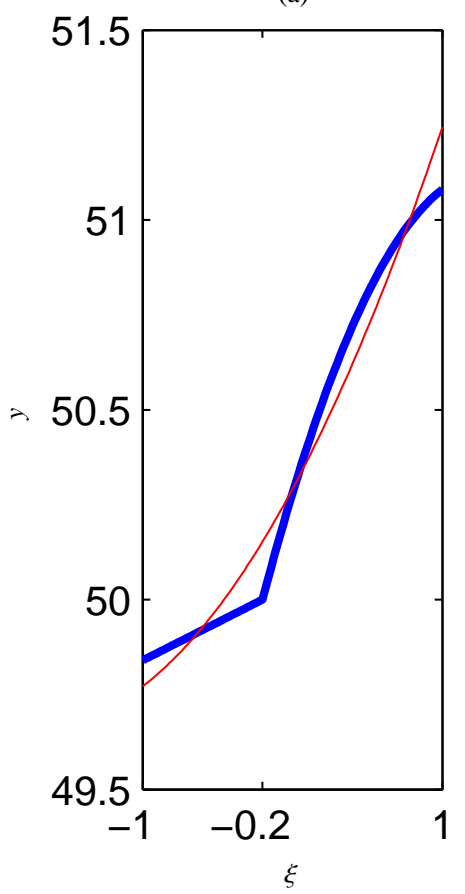

(b)

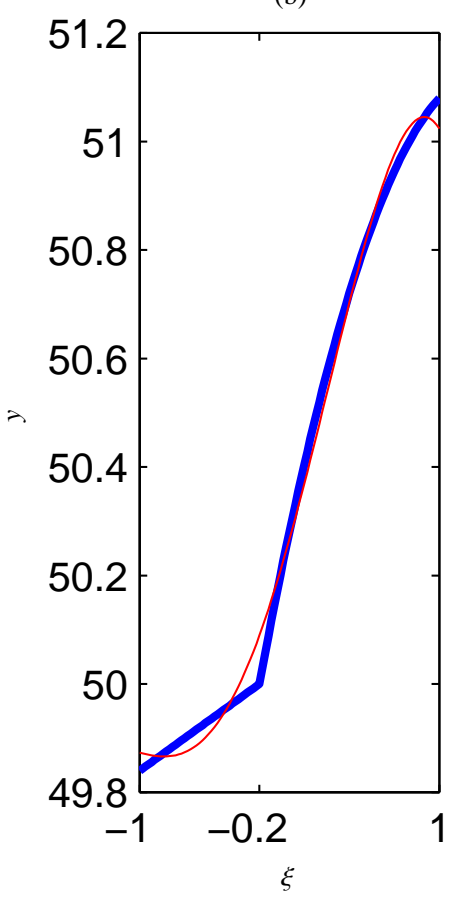

(c)

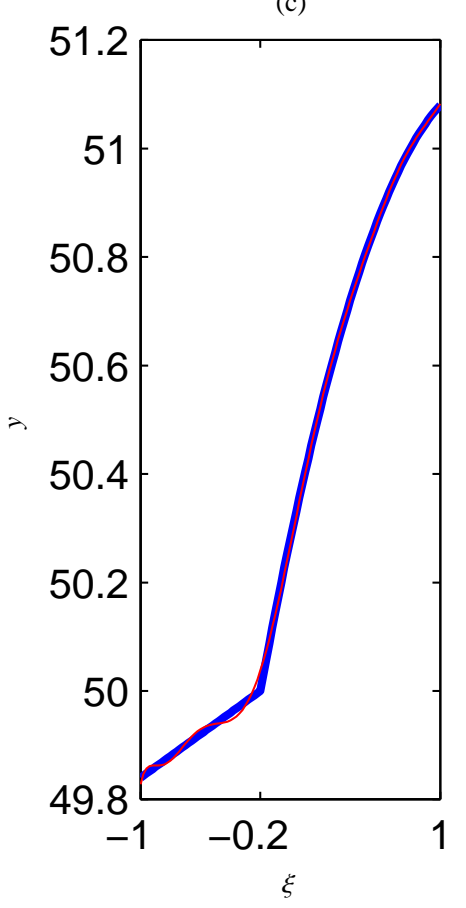

Figure 1: Limitations of a simple polynomial approximation of a target curve: - target curve; $\longrightarrow$ polynomial approximation $y=\psi(\xi)$ where $\psi$ has degree D. (a) $D=2$, (b) $D=4$, (c) $D=10$.

Separating real and imaginary parts of these equations, one gets $2 N(2 n+1)$ real scalar equations for $2 N\left(P_{k}+1\right)$ real unknowns. In case of a deterministic mode set $\mathcal{P}_{k}$ which does not include all the deterministic modes $\left(P_{k} \neq 2 n\right)$, this system is overconstrained. To get a square system, the final step is to pre-multiply Eqs. (12) by the transpose of the matrix containing the deterministic modes in $\mathcal{P}_{k}$. This finally leads to a square non-linear (quadratic) system with size $2 N\left(P_{k}+1\right)$ that can be processed using a dedicated non-linear solver.

This procedure lets us approximate eigenmodes $\tilde{\lambda}_{k}$ and $\tilde{\mathbf{u}}_{k}$ by a complex sum of multivariate polynomials $\boldsymbol{\psi}_{n}$. The usual statistical estimators such as mean and standard deviation can then be quickly evaluated over a large sample of $\boldsymbol{\xi}$ points by using formulas (8) and (11). This is equivalent to a Monte Carlo simulation but without the resolution of an eigenvalue problem for each realization and is much faster.

As this is a polynomial approximation, one needs to use high degrees to approximate quantities with strong variations accurately. For example, if an equilibrium becomes unstable over the input range under study, the first derivative of some eigenvalue may not be continuous; this makes the polynomial approximation very poorly appropriate to describe the eigenvalue evolution. From a more general point of view, studying changes in stability, it is obvious that different evolutions will be observed over the different stable and unstable ranges. This is illustrated on Fig. 1. On this figure, the target curve has a discontinuous derivative and $y$ axis matches for example the evolution of the imaginary part of an eigenvalue whose mode undergoes a change in stability for $\xi=-0.2$. The several polynomial approximations using different polynomial degrees displayed in Fig. 1 show that a high degree (pane (c) degree 10) is required to describe the change in slope accurately. Decompositions using a polynomial basis with such a high degree generate very large systems (12) and (13) to solve. To overcome this problem, one may use a piecewise polynomial approximation. The next section is devoted to the introduction of this piecewise approach into the general method.

\subsection{Working with a partitioned stochastic space}

The idea of using piecewise polynomial approximation of uncertain quantities has already been developed in the field of fluid mechanics [41, 42] for the study of limit cycles or equilibria. The subsequent method is referred to as Multi-Element generalized Polynomial Chaos (MEgPC). These studies use a decomposition of the random space to which the random variable vector $\boldsymbol{\xi}$ belongs. A polynomial approximation is then computed over each element of this decomposition. It is generally developed considering uniform laws for the random variable $\boldsymbol{\xi}$ which leads to using Legendre polynomials for the approximation. This restriction to a uniform law can be alleviated as non-uniform random variables can be approximated by a sum of Legendre polynomials of uniform random variables [41, 34].

In this section, the decomposition of the random space and its consequences on the stochastic eigenmodes approximation are exposed with a non-probabilistic point of view: it is presented as a piecewise polynomial fitting of eigenvalues and eigenvectors components depending on a vector of $Q$ real variables grouped in vector $\boldsymbol{\xi}$. The aim of the procedure is then to get the expressions of eigenmodes using a decomposition on a Legendre orthogonal polynomial basis on each element of the partition. Different statistical laws for uncertain input description are then handled by adapting the sample used for the Monte Carlo simulation. This MCS is expected to be realized quickly as the eigenmodes are evaluated using the piecewise 
polynomial approximation rather than by using a direct resolution of the eigenvalue problem.

One could object that the Legendre polynomial basis is not the one that suits best the input distribution (for example Gamma distribution) and hence, that the weight function used in the associated scalar product does not put stress on the most probable values. This is true except if the resulting description is accurate enough whatever the chosen point may be.

First the description of a quantity depending on $\boldsymbol{\xi}$ belonging to $S_{i}$, a bounded interval of $\mathbb{R}^{\mathrm{Q}}$ using a basis of Legendre multivariate polynomials depending on a variable $\zeta$ belonging to $\mathcal{I}^{Q}$ is exposed. Then, the problem of switching from such a description over $S_{i}$ to the description over $S_{j}$, included in $S_{i}$ is addressed. The global scheme using these fundamental steps is presented in Section 2.4.

Let $\boldsymbol{\psi}_{n}(\zeta), 1 \leq n \leq N$ be the Legendre multivariate polynomials basis with degree less or equal to $D$ in the case of $Q$ variables, defined over $\mathcal{I}^{Q}, \mathcal{I}=[\alpha, \beta]$. The scalar product associated is

$$
<f, g>=\int_{I Q} f(\boldsymbol{\zeta}) g(\boldsymbol{\zeta}) w(\boldsymbol{\zeta}) \mathrm{d} \boldsymbol{\zeta}
$$

where $w=1$ denotes the weight function associated to this polynomials family.

Let $S_{i}$ be a bounded interval of $\mathbb{R}^{\mathrm{Q}}$ and denote $\boldsymbol{v}_{i}$ the diffeomorphism mapping $S_{i}$ into $\mathcal{I}^{Q}$ :

$$
\begin{aligned}
\boldsymbol{v}_{i}: S_{i} & \rightarrow \mathcal{I}^{Q} \\
\boldsymbol{\xi} & \mapsto \boldsymbol{v}_{i}(\boldsymbol{\xi})=\boldsymbol{\zeta}
\end{aligned}
$$

If $S_{i}=\left[a_{1}^{(i)}, b_{1}^{(i)}\right] \times \cdots \times\left[a_{Q}^{(i)}, b_{Q}^{(i)}\right]$, one will use

$$
\forall k \in \llbracket 1, Q \rrbracket, \zeta_{k}=\frac{\alpha b_{k}^{(i)}-\beta a_{k}^{(i)}}{b_{k}^{(i)}-a_{k}^{(i)}}+\frac{\beta-\alpha}{b_{k}^{(i)}-a_{k}^{(i)}} \xi_{k}
$$

as $\boldsymbol{v}_{i}$ definition.

If $f$ is a scalar function of $\xi$ defined over $S$ and $\left\{S_{i}\right\}_{i=1 \ldots N_{S}}$ is a partition of $S$, its value over $S_{i}$ will then be computed using:

$$
f(\boldsymbol{\xi})=\sum_{n=1}^{N} f_{n}^{(i)} \boldsymbol{\psi}_{n}\left(\boldsymbol{v}_{i}(\boldsymbol{\xi})\right)
$$

The $f_{n}^{(i)}$ are the coefficients of the decomposition of $f$ on the polynomial basis $\boldsymbol{\psi}_{n}$ over $S_{i}$.

Considering the eigenvalue problem at stake, on will solve the following problem on each subset $S_{i}$ :

$$
\begin{gathered}
\tilde{\mathbf{A}}^{(i)} \tilde{\mathbf{u}}_{k}^{(i)}=\tilde{\lambda}_{k}^{(i)} \tilde{\mathbf{B}}^{(i)} \tilde{\mathbf{u}}_{k}^{(i)} \\
\left(\tilde{\mathbf{u}}_{k}^{(i)}\right)^{\mathrm{T}} \tilde{\mathbf{B}}^{(i)} \tilde{\mathbf{u}}_{k}^{(i)}=1
\end{gathered}
$$

with the following notations for each quantity involved:

$$
\begin{gathered}
\tilde{\mathbf{A}}^{(i)}(\boldsymbol{\xi})=\sum_{n=1}^{N_{A}} \mathbf{A}_{n}^{(i)} \boldsymbol{\psi}_{n}\left(\boldsymbol{v}_{i}(\boldsymbol{\xi})\right) \\
\tilde{\mathbf{B}}^{(i)}(\boldsymbol{\xi})=\sum_{n=1}^{N_{B}} \mathbf{B}_{n}^{(i)} \boldsymbol{\psi}_{n}\left(\boldsymbol{v}_{i}(\boldsymbol{\xi})\right) \\
\tilde{\lambda}_{k}^{(i)}(\boldsymbol{\xi})=\lambda_{k} \sum_{n=1}^{N}\left({ }^{(k)} a_{n}^{(i)}+\mathrm{j}^{(k)} b_{n}^{(i)}\right) \boldsymbol{\psi}_{n}\left(\boldsymbol{v}_{i}(\boldsymbol{\xi})\right) \\
\tilde{\mathbf{u}}_{k}^{(i)}(\boldsymbol{\xi})=\sum_{p \in \mathcal{P}_{k}}\left(\sum_{n=1}^{N}\left({ }^{(k)} \lambda_{n}^{p(i)}+\mathrm{j}^{(k)} \mu_{n}^{p(i)}\right) \boldsymbol{\psi}_{n}\left(\boldsymbol{v}_{i}(\boldsymbol{\xi})\right)\right) \mathbf{u}_{p}
\end{gathered}
$$

To get the coefficients $\mathbf{A}_{n}^{(i)}$ and $\mathbf{B}_{n}^{(i)}$, one must be able to deduce the decomposition of any quantity over an interval $S_{j}$ included in $S_{i}$ knowing the decomposition over $S_{i}$. This procedure is explained in the case of a scalar quantity $f$. It is easily adaptable to non-scalar quantities.

Let $S_{j}$ be an interval included into $S_{i}$ and $g$ the restriction of $f$ over this interval. $g$ decomposition over the $\boldsymbol{\psi}_{n}$ family is denoted:

$$
g(\boldsymbol{\xi})=\sum_{n=1}^{N} f_{n}^{(j)} \boldsymbol{\psi}_{n}\left(\boldsymbol{v}_{j}(\boldsymbol{\xi})\right)
$$


Knowing the coefficients $f_{n}^{(i)}$, one can compute the $f_{n}^{(j)}$ coefficients in the following manner: first, consider $\xi \in S_{j}$ and look for decomposition coefficients $\chi_{n, k}$ such that

$$
\boldsymbol{\psi}_{n}\left(\boldsymbol{v}_{i}(\boldsymbol{\xi})\right)=\sum_{k=1}^{N} \chi_{n, k} \boldsymbol{\psi}_{k}\left(\boldsymbol{v}_{j}(\boldsymbol{\xi})\right)
$$

The $\chi_{n, k}$ coefficients are found by solving for each $1 \leq n \leq N$ the system:

$$
\left[\begin{array}{c}
\boldsymbol{\psi}_{1}\left(\boldsymbol{v}_{j}\left(\boldsymbol{\xi}^{1}\right)\right) \ldots \boldsymbol{\psi}_{N}\left(\boldsymbol{v}_{j}\left(\boldsymbol{\xi}^{1}\right)\right) \\
\vdots \\
\boldsymbol{\psi}_{1}\left(\boldsymbol{v}_{j}\left(\boldsymbol{\xi}^{N}\right)\right) \ldots \boldsymbol{\psi}_{N}\left(\boldsymbol{v}_{j}\left(\boldsymbol{\xi}^{N}\right)\right)
\end{array}\right]\left\{\begin{array}{c}
\chi_{n, 1} \\
\vdots \\
\chi_{n, N}
\end{array}\right\}=\left\{\begin{array}{c}
\boldsymbol{\psi}_{n}\left(\boldsymbol{v}_{i}\left(\boldsymbol{\xi}^{1}\right)\right) \\
\vdots \\
\boldsymbol{\psi}_{n}\left(\boldsymbol{v}_{i}\left(\boldsymbol{\xi}^{N}\right)\right)
\end{array}\right\}, \boldsymbol{\xi}^{p} \in S_{j}
$$

That is, $N$ linear systems with size $N$ are solved to establish the $\chi$ matrix such that:

$$
\forall \xi \in S_{j},\left\{\begin{array}{c}
\boldsymbol{\psi}_{1}\left(\boldsymbol{v}_{i}(\xi)\right) \\
\vdots \\
\boldsymbol{\psi}_{N}\left(\boldsymbol{v}_{i}(\xi)\right)
\end{array}\right\}=\chi\left\{\begin{array}{c}
\boldsymbol{\psi}_{1}\left(\boldsymbol{v}_{j}(\xi)\right) \\
\vdots \\
\boldsymbol{\psi}_{N}\left(\boldsymbol{v}_{j}(\xi)\right)
\end{array}\right\}
$$

Finally, the $f_{n}^{(j)}$ coefficients are obtained from the $f_{n}^{(i)}$ using

$$
\left[f_{1}^{(j)}, \ldots, f_{N}^{(j)}\right]=\left[f_{1}^{(i)}, \ldots, f_{N}^{(i)}\right] \chi
$$

\subsection{The global algorithm: two possible strategies}

To present the global algorithm, we assume that the random inputs are described using $Q$ uniform random variables $\xi_{1}, \ldots, \xi_{Q}$ that vary over $S$, a bounded interval of $\mathbb{R}^{\mathrm{Q}}$. The aim of the algorithm is to return a collection of polynomial approximations of eigenmodes using a Legendre basis with $N$ elements over a partition of $S$ whose elements are denoted $S_{i}$; elements of the partition will be referred to as "cells". Over each $S_{i}$, one then has to determine the coefficients ${ }^{(k)} \lambda_{n}^{p(i)},{ }^{(k)} \mu_{n}^{p(i)}$, ${ }^{(k)} a_{s}^{(i)}$ and ${ }^{(k)} b_{s}^{(i)}$. For notation convenience, these $2 N\left(P_{k}+1\right)$ scalar coefficients are gathered in a vector denoted $\mathbf{x}^{(i)}$.

Two strategies to process the stochastic space are deployed and will be compared in Section 3: the first one relies on the dichotomy method and is close to the method proposed in [41, 42]. This method is exposed in Section 2.4.1. The second one invokes continuation principles and is presented in Section 2.4.2.

That said, the two strategies share a common scheme presented in Fig. 2. First, the algorithm is initialized with the set of matrices $\mathbf{A}_{n}$ and $\mathbf{B}_{n}$ that define the problem over $S$ and a first guess for decomposition coefficients $\mathbf{x}_{0}$; this vector can for example be chosen such that the processed stochastic eigenmode matches the deterministic mode over the whole $S$ interval. Then, the general loop is applied: first, if all the space $S$ has been processed, algorithm stops. Otherwise, a cell $S_{i}$ is defined in the remaining space as well as a startpoint $\mathbf{x}_{i}^{(i)}$, depending on the chosen method. The collection of matrices $\mathbf{A}_{n}^{(i)}$ and $\mathbf{B}_{n}^{(i)}$ defining the problem over the current cell are evaluated using (26) and (27) and the problem (12) and (13) is then solved, returning a new set of coefficients $\mathbf{x}_{f}^{(i)}$. Accuracy of the decomposition is tested as detailed in Section 2.4.3. If it satisfies the criterion or the criterion is not satisfied but no more cell can be created (a maximum computation time, a maximum number of cells or a minimum cell width has been reached), each element is stored (that is the current cell definition, eigenmode decomposition coefficients, matrices decomposition and accuracy criterion result). Otherwise, the current cell $S_{i}$ is refined accordingly to the chosen method.

\subsubsection{First method: Dichotomy}

The simplest way to refine a cell over which the decomposition is not satisfying is to split it in half in one direction to generate two smaller sub-cells. Then, a startpoint has to be chosen for future processing of each of these sub-cells; different choices are possible for this startpoint. First, one can always use the same startpoint $\mathbf{x}_{0}$ that is provided by the user to initialize the algorithm. Another possibility is to convert the final decomposition $\mathbf{x}_{f}^{(i)}$ over $S_{i}$ into equivalent decompositions over each of the sub-cell using formulas (26) and (27). Finally, one can extrapolate a decomposition from neighboring cells: considering a one dimensional problem, once the decomposition over one cell is validated, the startpoint for each of the two neighbors is the decomposition that matches constant values for eigenmodes over each sub-cell. The value is equal to the value of eigenmodes at the validated cell border. Fig. 3 illustrates this procedure: once the decomposition over $S_{i}$ is validated, each $\mathbf{x}^{(i)}$ component $x_{j}$ of neighboring cells $S_{\text {left }}$ ans $S_{\text {right }}$ is initialized with the value at corresponding border of $S_{i}$. This procedure giving better results than the two previous ones mentioned above, it is the one that is used for numerical applications of Section 3 . 


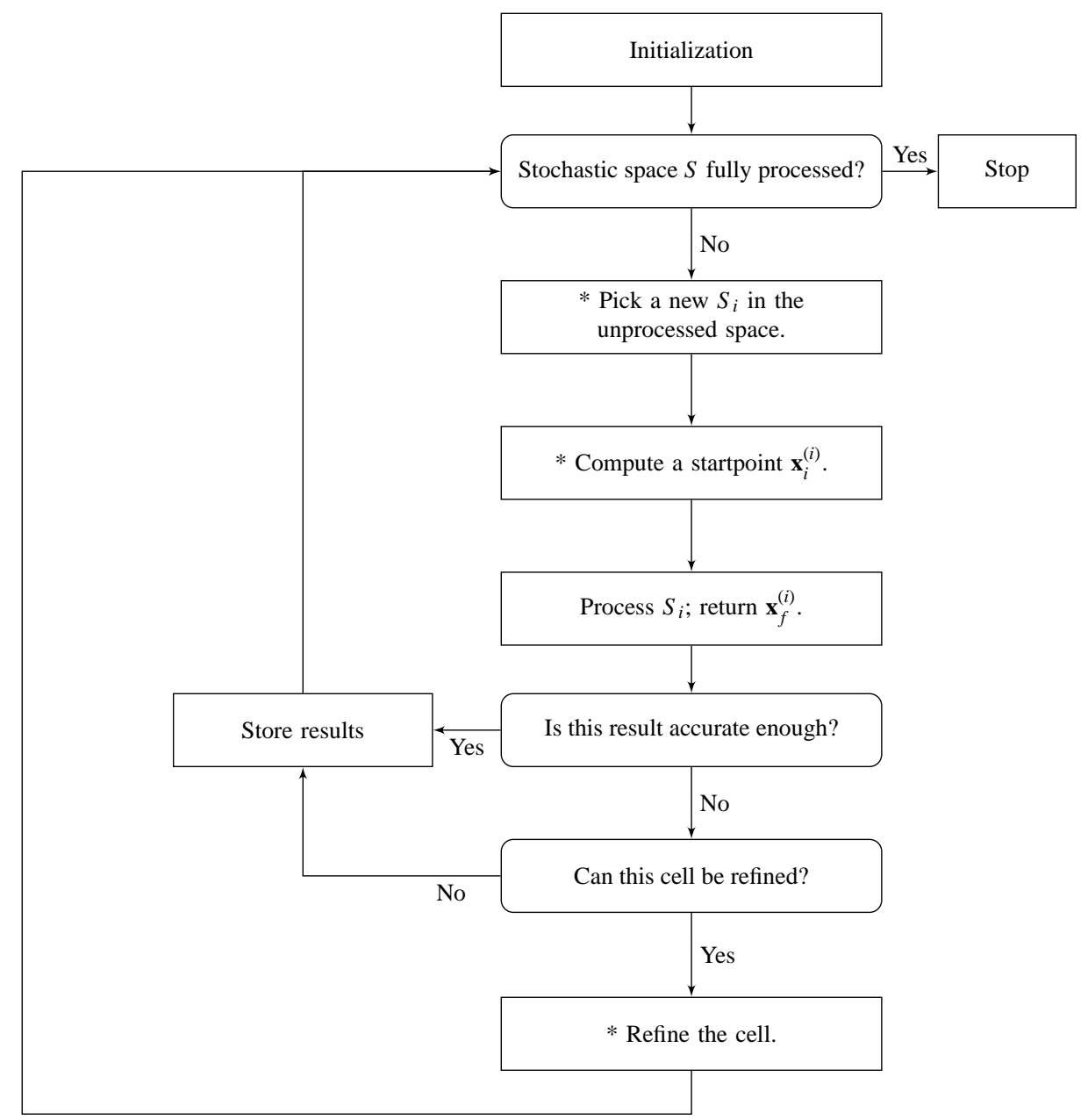

Figure 2: Global algorithm flowchart. Steps implying different processes for the method relying on an dichotomy approach and the one taking advantage of continuation principles are starred.

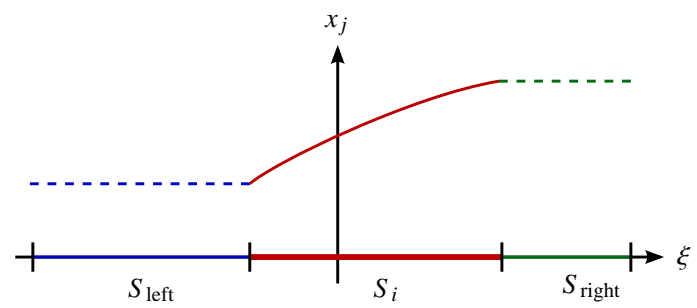

Figure 3: Method based on dichotomy: startpoint initialization strategy for cells $S_{\text {left }}$ and $S_{\text {right }}$ once decomposition over a cell $S_{i}$ is validated; $x_{j}$ is the $j$-th component of the vector of unknowns containing the eigenmode decomposition coefficients. 


\subsubsection{Second method: Continuation}

Another point of view is to consider that the sought values are continuous from one cell to another and hence, to apply some continuations principles (see [43] for the continuation methods). Hence, in one dimension, starting from the center of the initial cell, new cells are built and processed by having $\xi$ varying continuously to the right and then from the center to the left. Once a cell has a proper decomposition (satisfying the accuracy criterion), another cell - contiguous - is computed: its width depends on the current cell width and various parameters such as the number of iterations used to converge to the decomposition over this cell. If only a few iterations were used, the new cell width is increased, if the number of iterations exceeds a certain threshold, then the width of the next cell is decreased; otherwise, it is unchanged. When the decomposition over the current cell is not satisfying, the cell width is decreased too.

The startpoint over each cell processed is computed using the contiguous cell decomposition which is extrapolated over this new cell. This method, compared to the one based on dichotomy injects more information at each cell by using extrapolation of the contiguous decomposition and using a more appropriate size for each cell. Its efficiency is tested in Section 3.

\subsubsection{Testing the accuracy of the decomposition}

Once the solution $\mathbf{x}_{f}^{(i)}$ has been evaluated for cell $S_{i}$, one needs to decide whether the polynomial approximation is satisfying or not. That is, one wants to test if it is accurate enough over $S_{i}$. As comparing value of eigenmodes returned by the approximation and a direct computation for several points over the interval would cost too much, one proposes an original criterion based on the Rayleigh ratio that is very cheap to evaluate.

Combining Eqs. (17) and (18), one gets the Rayleigh ratio

$$
\forall \xi \in S_{i}, \frac{\left(\tilde{\mathbf{u}}_{k}^{(i)}\right)^{\mathrm{T}} \tilde{\mathbf{A}}^{(i)} \tilde{\mathbf{u}}_{k}^{(i)}}{\left(\tilde{\mathbf{u}}_{k}^{(i)}\right)^{\mathrm{T}} \tilde{\mathbf{B}}^{(i)} \tilde{\mathbf{u}}_{k}^{(i)}}=\tilde{\lambda}_{k}^{(i)}
$$

As the equations are satisfied in a weak sense only (only their projection over the polynomial basis are considered), this ratio may not be verified exactly. This provides a criterion to estimate the quality of the current decomposition by testing

$$
\epsilon_{k}(\boldsymbol{\xi})=\left|\left(\frac{\left(\tilde{\mathbf{u}}_{k}^{(i)}\right)^{\mathrm{T}} \tilde{\mathbf{A}}^{(i)} \tilde{\mathbf{u}}_{k}^{(i)}}{\left(\tilde{\mathbf{u}}_{k}^{(i)}\right)^{\mathrm{T}} \tilde{\mathbf{B}}^{(i)} \tilde{\mathbf{u}}_{k}^{(i)}}\right) / \tilde{\lambda}_{k}^{(i)}-1\right|
$$

for several points $\boldsymbol{\xi}$ in $S_{i}$.

\section{Application to a finite element model for brake squeal}

This section is devoted to the application of the proposed method to a brake model in order to conduct a stochastic study of brake squeal noise. First, the considered model is exposed along with the uncertain configurations. Then, the accuracy of the method using successively a dichotomy and a continuation approach is evaluated through the comparison of their results to direct resolutions. Finally, histograms are built, based on these results in order to support the stochastic study of the system and demonstrate its efficiency compared to Monte Carlo simulations.

\subsection{Brake model and uncertain configurations}

The model used is derived from the finite element model described in [13, 44]. It consists of a rotating disc and a fixed pad as displayed in Fig. 4. The disc and the pad are modeled using the finite element method. Each model is reduced using a Craig and Bampton technique: 4 contact nodes are kept on each part (pad and disc) as well as 4 dofs (degrees of freedom) on the backplate of the pad for application of hydraulic pressure; the first 30 eigenmodes are retained on each part. Such a reduction may degrade the pertinence of the results regarding the real behavior of the brake system. This problem should be addressed carefully in the frame of a study which aims at getting quantitative results. The current model, used in previously cited work, was provided as is to the authors. The assembled model has then 88 dofs and the set of equations can be written

$$
\mathbf{M} \ddot{\mathbf{q}}+\mathbf{D} \dot{\mathbf{q}}+\mathbf{K q}+\mathbf{K}_{\mathrm{c}} \mathbf{q}=\mathbf{f}_{\mathrm{e}}
$$

where $\mathbf{M}$ is the mass matrix, $\mathbf{K}$ is the structural stiffness matrix, $\mathbf{D}$ is the damping matrix with a Rayleigh expression $\mathbf{D}=$ $\alpha \mathbf{M}+\beta \mathbf{K}, \mathbf{K}_{\mathrm{c}}$ takes into account contact forces acting between the pad and the disc and $\mathbf{f}_{\mathrm{e}}$ stands for the force due to brake pressure applied on the pad. $\mathbf{K}_{\mathrm{c}}$ is a non-symmetric matrix that couples the two subsystems - the pad and the disc. It consists in the addition of two matrices: a first one, symmetric, which represents the normal force at the contact surface and which is proportional to the contact stiffness denoted $k_{c}$. The second matrix that contributes to $\mathbf{K}_{\mathrm{c}}$ is non-symmetric and stands for the tangent force at the contact interface; it is proportional to the product of the friction coefficient - denoted $\mu$ - and $k_{c}$. The contact follows a Coulomb friction law with constant friction coefficient $\mu$ and assumption of permanent sliding is made; more complex phenomenon such as stick-slip or sprag-slip are then put aside. Normal and tangential contact forces applied by the pad over the disc are opposite to forces applied by the disc on the pad and can be formulated as follows for contact node $i, 1 \leq i \leq 4$ :

$$
F_{i, \text { normal }}=k_{c}\left(U_{i, \text { Pad }}-U_{i, \text { Disc }}\right)
$$




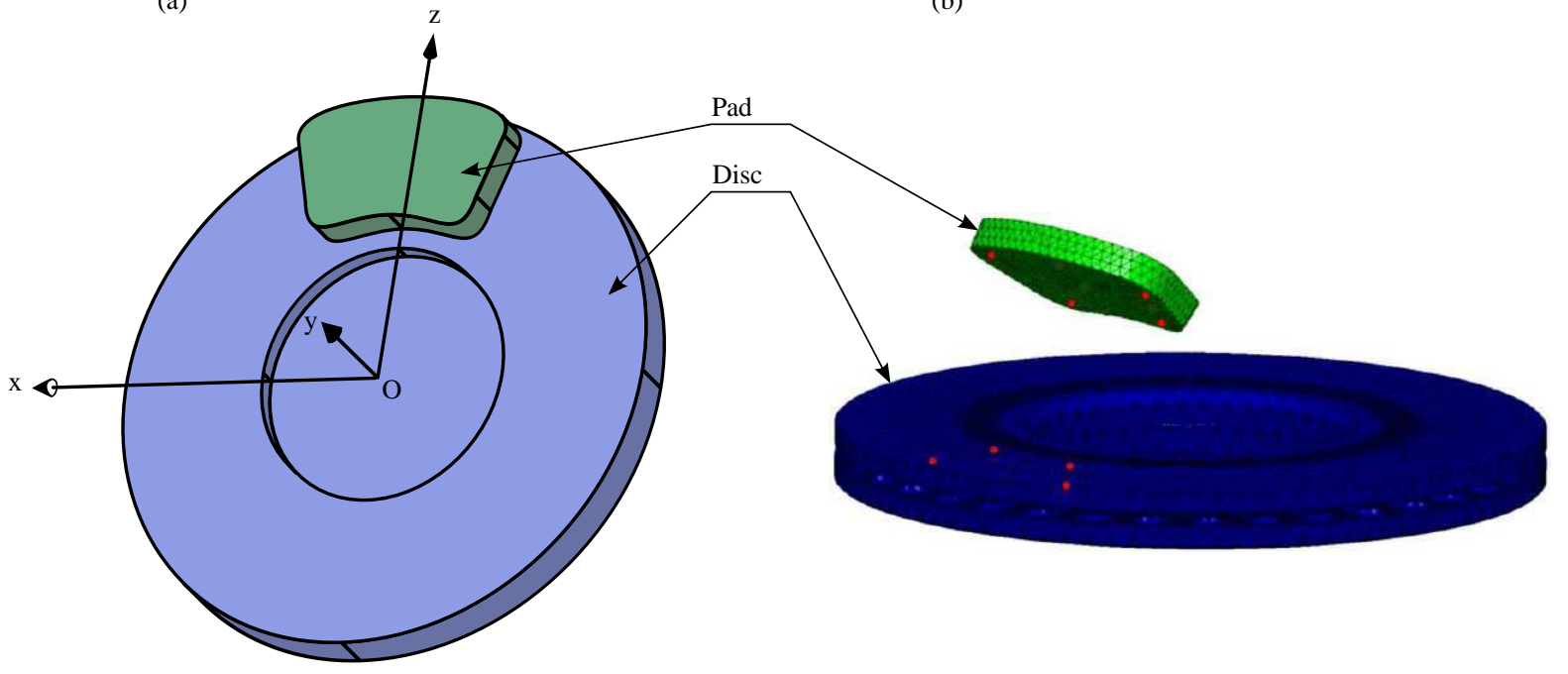

Figure 4: Brake model. (a) Plan; (b) FE model.

$$
F_{i, \text { tangent }}=\mu F_{i, \text { normal }} \operatorname{sign}\left(\mathrm{V}_{\mathrm{i}, \text { rel }}\right)
$$

where $U_{i, \mathrm{Pad} / \mathrm{Dic}}$ and $V_{i \text {,rel }}$ denotes the normal displacement of node $i$ for the pad or the disc and the relative velocity between the pad and the disc at contact node $i$ respectively.

This coupling of the two substructures affects both lower and higher frequency modes as shown by Fig. 5. The upper part (a) of this figure displays the absolute difference between the Modal Assurance Criterion (MAC, [45]) of the undamped uncoupled system compared to itself and the MAC of the undamped coupled system compared to the uncoupled eigenvectors (see Eq. (33)).

$$
\Delta \mathrm{MAC}=\left|\operatorname{MAC}\left(\mathbf{V}_{\text {uncoupled }}, \mathbf{V}_{\text {coupled }}\right)-\operatorname{MAC}\left(\mathbf{V}_{\text {uncoupled }}, \mathbf{V}_{\text {uncoupled }}\right)\right|
$$

Higher values of this difference let us detect the modes that are most affected by the coupling of pad and disc structures. One considers here that the $j$-th mode is significantly modified if one of the terms of the $j$-th column of $\triangle \mathrm{MAC}$ is greater than 0.5. The lower part (b) then outlines eigenfrequencies of the coupled system that match these modes using red dots. Values used for this comparison are $k_{c}=1 \cdot 10^{7} \mathrm{~N} . \mathrm{m}^{-1}$ and $\mu=0.25$.

As recalled in Section 2.1, the stability of any static equilibrium of Eq. (30) is determined by the following tangent problem:

$$
\mathbf{M} \ddot{\mathbf{r}}+\mathbf{D} \dot{\mathbf{r}}+\left(\mathbf{K}+\mathbf{K}_{\mathrm{c}}\right) \mathbf{r}=\mathbf{0}
$$

One will then focus on the eigenvalues of this tangent system while including some uncertain parameters.

Two nominal configurations are studied. A first one with a contact stiffness $k_{c}=1 \cdot 10^{7} \mathrm{~N} . \mathrm{m}^{-1}$ and a second one with a contact stiffness twice larger $\left(k_{c}=2 \cdot 10^{7} \mathrm{~N} . \mathrm{m}^{-1}\right)$. These two configurations correspond to a tangent linear contact state obtained by experimental tests of pad compression [44]. For each configuration, the variation of the friction coefficient $\mu$ or the contact stiffness $k_{c}$ is evaluated. Uncertainty is introduced as follows:

$$
\mu(\xi)=\mu_{0}(1+\xi \Delta \mu)
$$

with $\mu_{0}=0.25$ and $\Delta \mu=0.5$, and

$$
k_{c}(\xi)=k_{c 0}\left(1+\xi \Delta k_{c}\right)
$$

with $k_{c 0}=1 \cdot 10^{7} \mathrm{~N} \cdot \mathrm{m}^{-1}$ or $k_{c 0}=2 \cdot 10^{7} \mathrm{~N} \cdot \mathrm{m}^{-1}$ and $\Delta k_{c}=0.5$.

$\xi$ is a random variable following a uniform law on $[-1,1]$. This means that for each configuration a variation of $\pm 50 \%$ of the parameter $\mu$ or $k_{c}$ is examined. These large variation ranges will let us test the robustness of the method.

Unstable modes evolution along with the random variable $\xi$ are displayed in Fig. 6 for configuration 2, case 2 ( $k_{c}$ varies). For each mode, both real and imaginary parts are plotted. This figure shows that the evolution of the studied modes can be quite complex and non-regular over the studied range. Configuration 1 and case 1 (when $\mu$ varies) would produce similar figures. Table 1 lists unstable areas per mode in the case when $\mu$ varies for each configuration and case.

\subsection{Algorithm accuracy}

The two versions of the procedure exposed in Section 2.4 were applied to each configuration for each case. Unstable modes were approximated one by one, using a Legendre polynomial basis with maximum degree $D=2$. As suggested in Section 2.3, only a few deterministic modes were retained to describe the stochastic modes. Based on the results of Fig. 5, only the most impacted modes were kept (modes 1 to 34,38 to 41 and 65 to 88 as well as their complex conjugates). This leads to a set $\mathcal{P}_{k}$ with $P_{k}=124$ modes out of 176 for each unstable stochastic mode $\tilde{\mathbf{u}}_{k}$. 
(a)
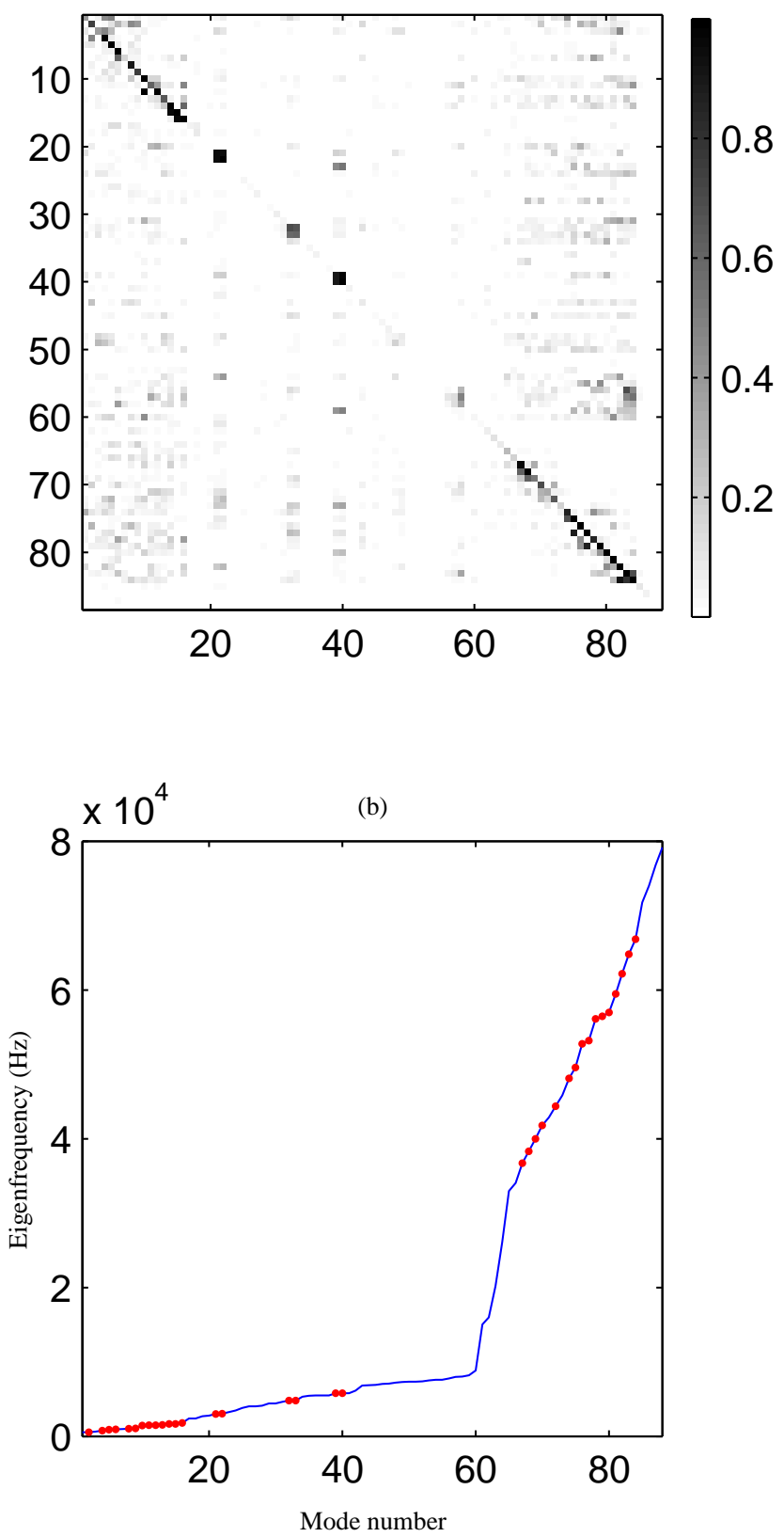

Figure 5: Modal modification due to the coupling. (a) Absolute difference between MAC matrices; (b) Eigenfrequencies of the coupled system: dots denote eigenfrequencies for eigenmodes whose MAC columns maximum absolute differences are greater than 0.5 .

Table 1: Unstable ranges per mode when $\mu$ varies for (a) configuration $1\left(k_{c}=1 \cdot 10^{7} \mathrm{~N} \cdot \mathrm{m}^{-1}\right)$ and (b) configuration $2\left(k_{c}=2 \cdot 10^{7} \mathrm{~N} \cdot \mathrm{m}^{-1}\right)$.

(a)

\begin{tabular}{lcc} 
Mode & $\mu$ & $\operatorname{Im}(\lambda)\left(\mathrm{rad.s}^{-1}\right)$ \\
\hline 6 & {$\left[0.30, \mu_{\max }\right]$} & {$\left[5.59 \cdot 10^{3}, 5.65 \cdot 10^{3}\right]$} \\
12 & {$\left[\mu_{\min }, 0.16\right]$} & {$\left[9.21 \cdot 10^{3}, 9.22 \cdot 10^{3}\right]$} \\
& {$\left[0.20, \mu_{\max }\right]$} & {$\left[9.25 \cdot 10^{3}, 9.48 \cdot 10^{3}\right]$} \\
69 & {$\left[\mu_{\min }, 0.13\right]$} & {$\left[2.47 \cdot 10^{5}, 2.47 \cdot 10^{5}\right]$} \\
78 & {$\left[0.26, \mu_{\max }\right]$} & {$\left[3.54 \cdot 10^{5}, 3.57 \cdot 10^{5}\right]$} \\
81 & {$\left[0.33, \mu_{\max }\right]$} & {$\left[3.78 \cdot 10^{5}, 3.79 \cdot 10^{5}\right]$}
\end{tabular}

(b)

\begin{tabular}{lcc} 
Mode & $\mu$ & $\operatorname{Im}(\lambda)\left(\mathrm{rad} . \mathrm{s}^{-1}\right)$ \\
\hline 6 & {$\left[0.29, \mu_{\max }\right]$} & {$\left[6.10 \cdot 10^{3}, 6.28 \cdot 10^{3}\right]$} \\
9 & {$\left[0.31, \mu_{\max }\right]$} & {$\left[6.41 \cdot 10^{3}, 6.74 \cdot 10^{3}\right]$} \\
13 & {$\left[0.29, \mu_{\max }\right]$} & {$\left[9.92 \cdot 10^{3}, 1.01 \cdot 10^{4}\right]$} \\
69 & {$\left[0.35, \mu_{\max }\right]$} & {$\left[2.63 \cdot 10^{5}, 2.63 \cdot 10^{5}\right]$} \\
73 & {$\left[0.35, \mu_{\max }\right]$} & {$\left[2.92 \cdot 10^{5}, 2.92 \cdot 10^{5}\right]$} \\
82 & {$\left[0.20, \mu_{\max }\right]$} & {$\left[4.06 \cdot 10^{5}, 4.06 \cdot 10^{5}\right]$} \\
86 & {$\left[0.27, \mu_{\max }\right]$} & {$\left[4.75 \cdot 10^{5}, 4.76 \cdot 10^{5}\right]$}
\end{tabular}


(a)
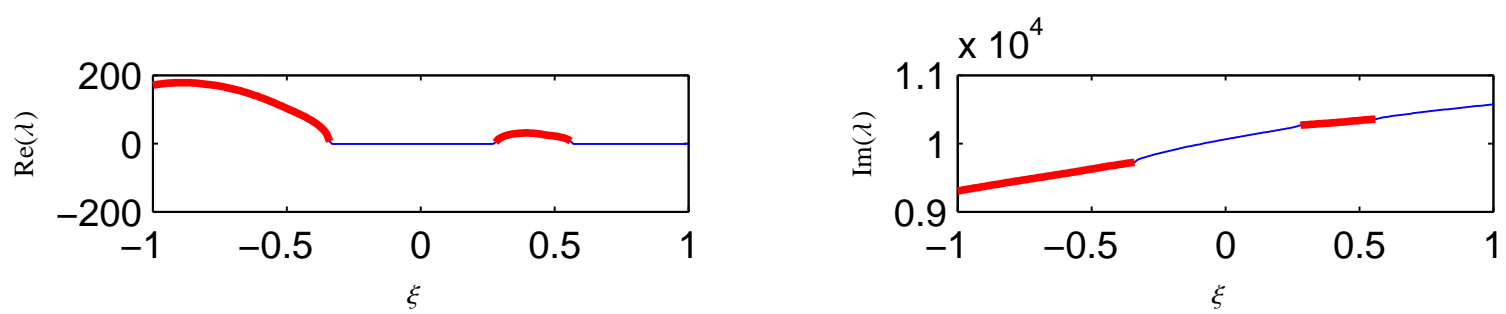

(b)
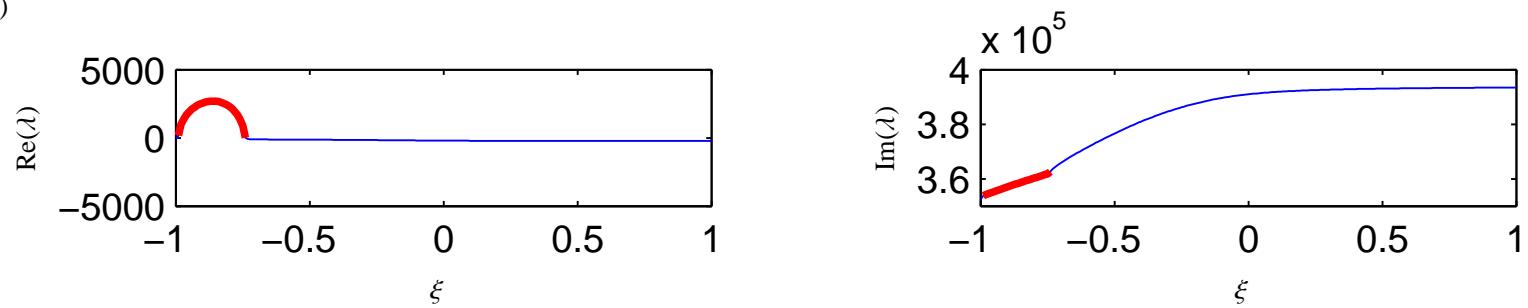

(c)
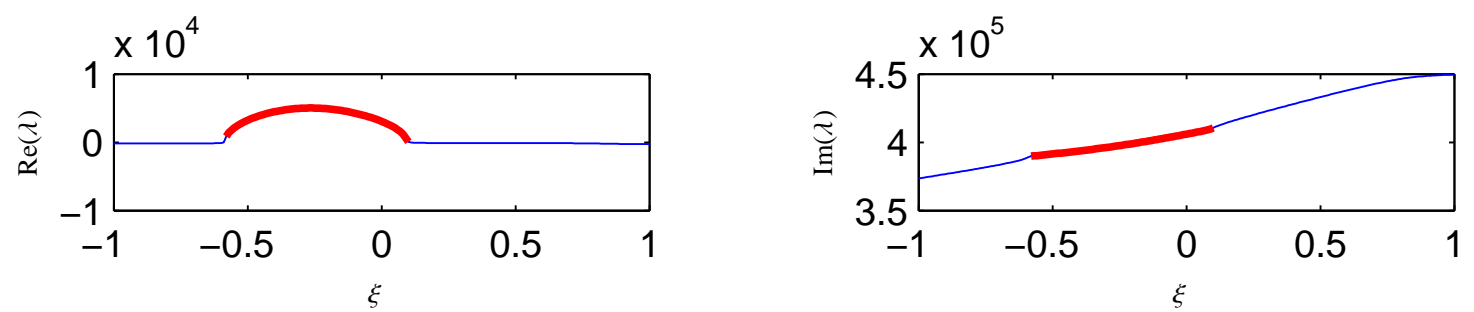

(d)
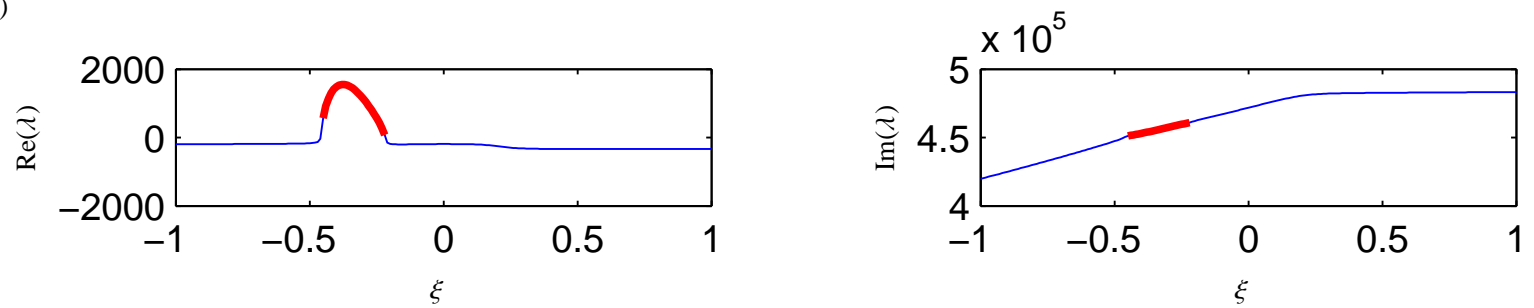

(e)
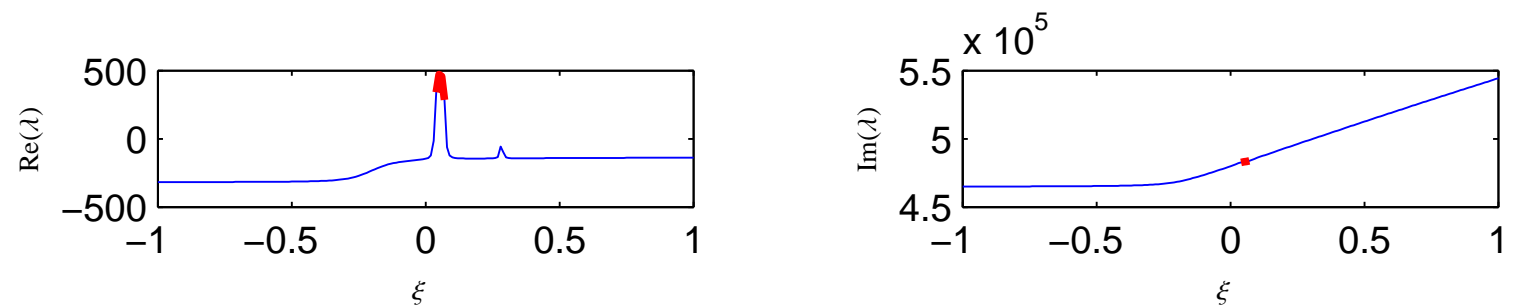

Figure 6: Unstable modes: Config $2\left(k_{c}=2 \cdot 10^{7} \mathrm{~N} \cdot \mathrm{m}^{-1}\right)$, Case 2 ( $k_{c}$ varies); Variation of complex eigenvalue $\lambda$ with random variable $\xi$ for (a) mode 13 , (b) mode 80 , (c) mode 82 , (d) mode 85 , (e) mode 86 ; — stable range, $\longrightarrow$ unstable range. 
Table 2: Accuracy of the method: Worst value over the unstable modes set for each configuration and case considering (a) method based on dichotomy and (b) method based on continuation principles.

(a)

\begin{tabular}{ccccc} 
& \multicolumn{2}{c}{ Configuration 1 } & \multicolumn{2}{c}{ Configuration 2 } \\
Indicator & Case 1 & Case 2 & Case 1 & Case 2 \\
\hline$\%\left(e_{\operatorname{Re} \lambda}<2 \%\right)$ & 97 & 76 & 91 & 75 \\
$\%\left(e_{\operatorname{Re} \lambda}<5 \%\right)$ & 98 & 97 & 97 & 97 \\
$\%\left(e_{\operatorname{Im} \lambda}<2 \%\right)$ & 100 & 87 & 100 & 97 \\
$\%\left(e_{\operatorname{Im} \lambda}<5 \%\right)$ & 100 & 100 & 100 & 100 \\
$\%\left(e_{\phi}<5 \%\right)$ & 100 & 78 & 100 & 78 \\
$\%\left(e_{\operatorname{sign}(\operatorname{Re} \lambda)}\right)$ & 0 & 0.5 & 0.5 & 0.5 \\
$e_{\xi \operatorname{Hopf}}$ & 0.00062 & 0.0097 & 0.0042 & 0.0059
\end{tabular}

(b)

\begin{tabular}{ccccc} 
& \multicolumn{2}{c}{ Configuration 1 } & \multicolumn{2}{c}{ Configuration 2 } \\
Indicator & Case 1 & Case 2 & Case 1 & Case 2 \\
\hline$\%\left(e_{\operatorname{Re} \lambda}<2 \%\right)$ & 97 & 94 & 91 & 97 \\
$\%\left(e_{\operatorname{Re} \lambda}<5 \%\right)$ & 97 & 97 & 97 & 99 \\
$\%\left(e_{\operatorname{Im} \lambda}<2 \%\right)$ & 100 & 100 & 100 & 100 \\
$\%\left(e_{\operatorname{Im} \lambda}<5 \%\right)$ & 100 & 100 & 100 & 100 \\
$\%\left(e_{\phi}<5 \%\right)$ & 100 & 100 & 100 & 100 \\
$\%\left(e_{\operatorname{sign}(\operatorname{Re} \lambda)}\right)$ & 0 & 0.5 & 0.5 & 0.5 \\
$e_{\xi \operatorname{Hopf}}$ & 0.00092 & 0.0097 & 0.0045 & 0.0012
\end{tabular}

Accuracy is evaluated by comparing results obtained using a direct evaluation and approximated ones over 201 points equally spaced over the range [-1,1] of the random variable $\xi$. To report the accuracy of the method, global indicators are proposed instead of local ones that would generate a long list of values considering the total number of unstable modes that have been approximated. One will focus on the relative error over the real and imaginary parts of eigenvalues, the error on the sign of the real part, the error on the Hopf bifurcation point localization (point at which the real part of an eigenvalue crosses the abscissa axis having a non-null imaginary part) and finally the error over the eigenvectors even if these are quantities of lesser interest for this study. The formulas for the first two relative error estimates are:

$$
\begin{aligned}
& e_{\operatorname{Re} \lambda}=\left|\frac{\operatorname{Re}\left(\lambda^{\mathrm{PC}}\right)-\operatorname{Re}\left(\lambda^{\mathrm{MC}}\right)}{\operatorname{Re}\left(\lambda^{\mathrm{MC}}\right)}\right| \times 100 \\
& e_{\mathrm{Im} \lambda}=\left|\frac{\operatorname{Im}\left(\lambda^{\mathrm{PC}}\right)-\operatorname{Im}\left(\lambda^{\mathrm{MC}}\right)}{\operatorname{Im}\left(\lambda^{\mathrm{MC}}\right)}\right| \times 100
\end{aligned}
$$

where PC and MC superscripts respectively denote results obtained using the approximation and a direct evaluation respectively. The error on the sign of the real part of eigenvalues is a binary indicator telling whether the sign is the same for both approximated and direct evaluation or not. The error on the Hopf bifurcation point localization is the distance between $\xi$ points for which the bifurcation occurs. This distance is to be compared to the width of the interval which is 2 . Finally, the relative error on eigenvectors is evaluated using the following formula which returns a scalar indicator for each $\xi$ point considered and each eigenvector:

$$
e_{\phi}=\max \left|\frac{\left|\mathbf{u}^{\mathrm{PC}}\right|-\left|\mathbf{u}^{\mathrm{MC}}\right|}{\max \left|\mathbf{u}^{\mathrm{MC}}\right|}\right| \times 100
$$

Rather than displaying these indicators over the [-1,1] range, one will give the percentage of points that are below $2 \%$ and $5 \%$ of error in case of relative errors on eigenvalues, below $5 \%$ for relative error on eigenvectors, the percentage of points for which an error on the sign of the real part is committed and finally the distance between $\xi$ points at bifurcations.

Finally, these results are summed up in Table 2 for each configuration and case by returning the worst result among the several modes. Obviously, both versions of the proposed method return very accurate results: stability is well evaluated (the percentage of points whose real part sign is wrong, $e_{\operatorname{sign}(\operatorname{Re} \lambda)}$, is lower than 0.5$)$ and Hopf bifurcation points are properly identified with errors $e_{\xi \text { Hopf }}$ lower than $1 \cdot 10^{-3}$ for an interval width equal to 2 . Moreover, errors on the eigenfrequencies $e_{\operatorname{Im} \lambda}$ are very limited too, all of the approximated values being equal to the exact ones more or less $5 \%$. Real parts of eigenvalues are also well estimated, particularly the one returned by the algorithm relying on continuation principles. Finally, it is worth to be noted that eigenvectors are estimated with a satisfying accuracy by the second method (based on continuation principles) especially, given that all the tested points present an overall error smaller than $5 \%$. If these quantities are not of the utmost importance in the context of a linear stability study, they may be useful when one aims at finding the limit cycle, providing a useful direction in which to look for it or, to analyze variations of mode shapes whether the system undergoes a bifurcation or not.

Considering this first set of results, both versions of the proposed method prove their ability to estimate eigenvalues of a uncertain system accurately, with a slight advantage for the version invoking continuation principles with a variable cell size and an extrapolation of neighboring cell result used as a startpoint. 


\subsection{Stochastic study and comments - Method efficiency}

Now that the method has been tested and showed a satisfying accuracy, this section is devoted to the stochastic analysis of the brake system. As one of the advantages of this general method is to provide a decomposition accurate whatever the input random law is, the decompositions of stochastic eigenmodes are used to test different random input laws for each case: based on experimental work [18] a uniform-like input law was designed to model friction coefficient variation. The following probability density function (pdf) over $[-1,1]$ was chosen to tend to reproduce the shape of experimental data available in this work:

$$
\begin{cases}f(\xi)=-10.0446 \xi^{2}-15.9375 \xi-5.8929 & \text { if }-1 \leq \xi<-0.8 \\ f(\xi)=0.1339(\xi+0.8)+0.4286 & \text { if }-0.8 \leq \xi<0.8 \\ f(\xi)=-16.7411 \xi^{2}+26.9196 \xi-10.1786 & \text { if } 0.8 \leq \xi \leq 1\end{cases}
$$

The continuous pdf is depicted in Fig. 7 (a), superimposed to the histogram describing the sample used for the simulation. This law is almost uniform, with a greater probability for higher values to occur. The subsequent results are compared to the ones obtained via a truncated Gaussian input law whose sample histogram is provided in Fig. 8:

$$
f(\xi)=\frac{1}{\sqrt{2 \pi \times 0.3^{2}}} \mathrm{e}^{-\frac{\xi^{2}}{2 \times 0.3^{2}}},-1 \leq \xi \leq 1
$$

For case 2, when the contact stiffness $k_{c}$ is uncertain, a uniform law, Fig. 9 (a.1) and (a.2), is compared to a truncated normal law (41) depicted in Fig. 10 (a.1) for configuration 1 and Fig. 10 (a.2) for configuration 2.

For each case and each law, the following results are given for configuration $1\left(k_{c}=1 \cdot 10^{7} \mathrm{~N} . \mathrm{m}^{-1}\right.$, pane (b) of Figs. 7 to 10$)$ and $2\left(k_{c}=2 \cdot 10^{7} \mathrm{~N} \cdot \mathrm{m}^{-1}\right.$, pane (c) of Figs. 7 to 10$)$ : first, the percentage of stable and unstable occurrences with specification of the number of unstable modes is displayed. This lets us analyze the propensity to squeal and the difficulty to prevent squealing (related to the number of unstable modes to stabilize). Second, an histogram depicting the unstable frequencies (that is the imaginary part of eigenvalues with positive real part) is provided. Finally, a third histogram displays the repartition of real parts of unstable modes. This gives information about the instability growth rate.

The results displayed in Figs. 7, 8, 9 and 10 come from the second version of the method, based on continuation principles which was proven to be more accurate. Nonetheless, the other version of the method was applied too so as to provide a comparison of numerical resources consumption which is addressed in the last part of this section.

Considering the first case relative to friction coefficient $\mu$ variation for both configurations and two different input laws whose results are depicted in Figs. 7 and 8, one can first denote that results notably vary when switching from a uniform-like input law to a truncated Gaussian one: while the simulation based on a truncated Gaussian law parameter variation returns a lower number of simultaneously unstable modes, it also decreases the number of stable occurrences for both configurations. Moreover, unstable frequencies in the range $[40,50] \mathrm{kHz}$ that appear in the case of simulations using a uniform-like law for the second configuration are not represented when considering a truncated normal distribution.

Similar remarks can be inferred from comparison of the second case simulations (when $k_{c}$ varies) relying on the one hand on a uniform law (Fig. 9) and on the other hand on a truncated Gaussian law (Fig. 10): the truncated Gaussian law returns more pessimistic results in terms of stability with respectively $0.6 \%$ and $22 \%$ of stable occurrences for configurations 1 and 2 to be compared to $7 \%$ and $30 \%$ in the case when a uniform distribution is used.

These comparisons of results based on the same decomposition of uncertain eigenvalues for different random laws emphasize the interest of the method that lets us reuse the decompositions computed once for all and hence save time compared to Monte Carlo simulations as will be discussed at the end of this section. Indeed, results returned when using several input laws are widely different and would merely not lead to the same optimization objectives in an engineering process. As the description of a random parameter such as friction coefficient may not be easy, this ability to quickly simulate a large panel of laws, including non-classical ones (such as the proposed uniform-like law) is a non-negligible advantage.

Comparing the results obtained for configuration $1\left(k_{c}=1 \cdot 10^{7} \mathrm{~N} \cdot \mathrm{m}^{-1}\right)$ and configuration $2\left(k_{c}=2 \cdot 10^{7} \mathrm{~N} \cdot \mathrm{m}^{-1}\right)$ for the same random law leads to the conclusion that a more stiff contact leads to a greater number of simultaneously unstable modes: up to 7 modes can become unstable simultaneously for configuration 2 (Figs. 7 (c) and 8 (c)) whereas a maximum of 4 modes is observed in the first configuration (Figs. 7 (b) and 8 (b)) when $\mu$ varies and these are 3 (Figs. 9 (c) and 10 (c)) against 2 (Figs. 9 (b) and 10 (b)) unstable modes for the second case studying variations of $k_{c}$. The number of stable occurrences merely increases with contact stiffness turning, for the first processed case, from $12.5 \%$ to $24 \%$ in the case of a uniform-like law for $\mu$ variation and from $7.6 \%$ to $9.8 \%$ in the case of a truncated Gaussian law. It is to be noted that a greater stiffness generates also a wider spectral range with higher frequencies (see "Unstable frequencies" histograms from panes (b) and (c) of Figs. 7 and 8). Finally, the real parts of unstable eigenvalues are increased too in configuration 2 which implies that instability develops itself quicker.

These sets of results tend to demonstrate that, for the studied system, a more stiff contact between the pad and the disc is more likely to lead to a stable brake behavior but that unstable ranges are harder to handle as a greater number of modes can be simultaneously unstable and hence optimization should take into account more constraints; moreover instability growth rate can become greater (larger positive real parts for eigenvalues).

The last part of this section is dedicated to the efficiency of the method compared to Monte Carlo simulations and the efficiency of each version of this method compared to the other. To this end, Table 3 summarizes CPU times for full simulations over a sample with size 1000 and 10000 . For each of the proposed method version, total duration $t_{\text {total }}$ is split into $t_{\text {fit }}$, that is the time needed for getting the decomposition and $t_{\text {shoot }}$ which is the amount of CPU seconds spent for evaluating the 1000 
(a)

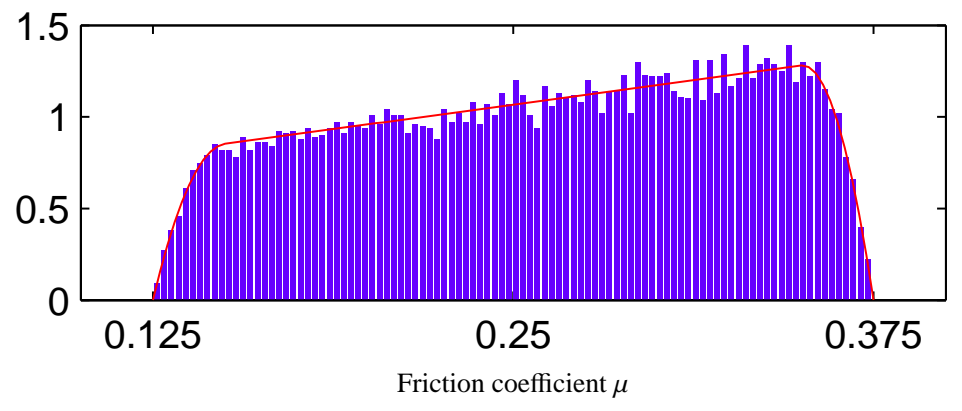

(b.1)

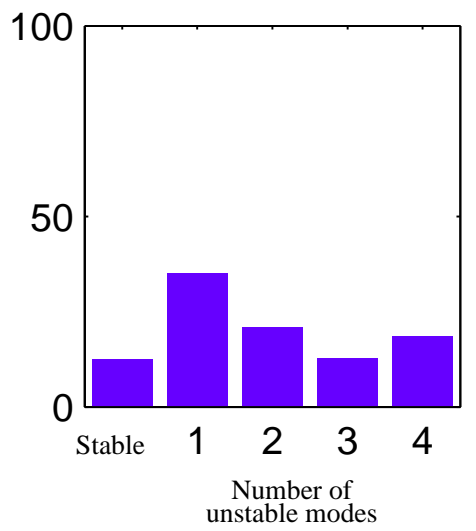

(c.1)

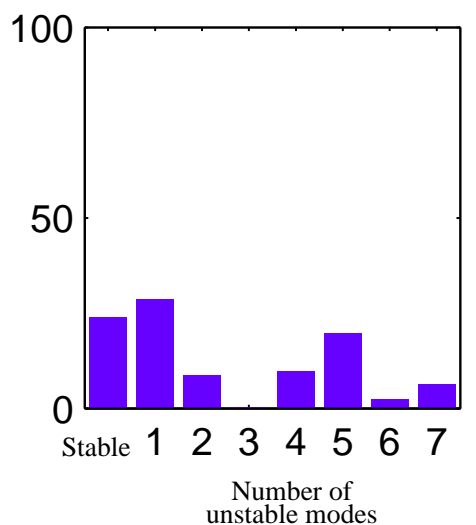

(b. 2$)$

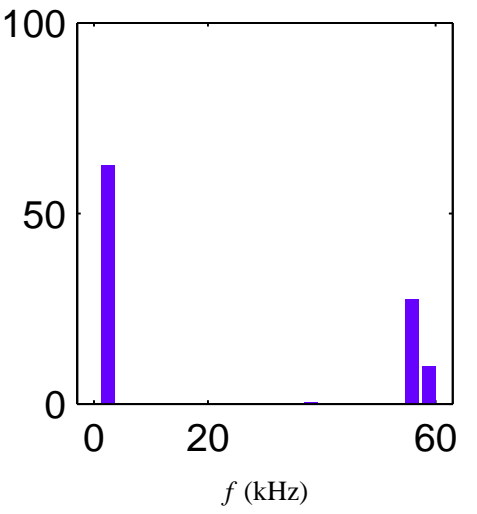

(c.2)

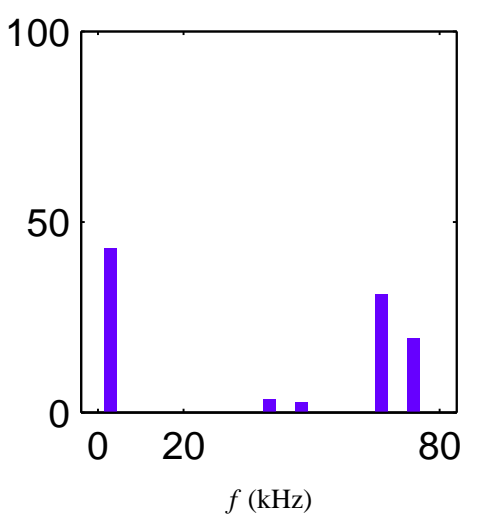

(b.3)

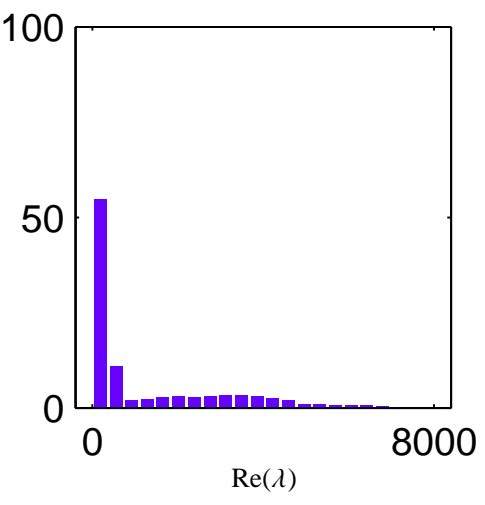

(c.3)

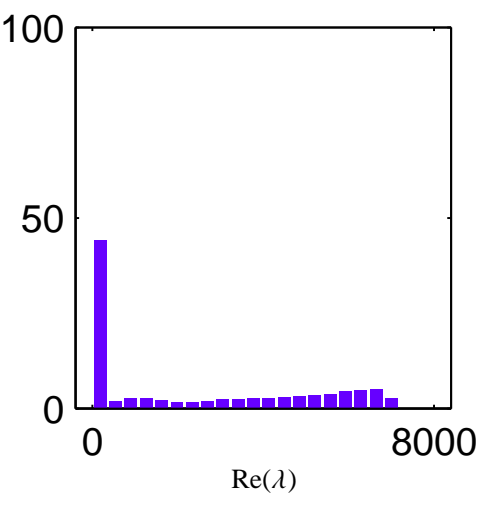

Figure 7: Simulations using a uniform-like law for case 1 ( $\mu$ varies): histograms. (a) Random parameter value occurrences $(\%)$; System stability and squeal information for (b.n) configuration 1 and (c.n) configuration 2; (b/c.1) Histogram of stable/unstable occurrences (\%), (b/c.2) Histogram of unstable frequencies $(\mathrm{kHz})$ occurrences $(\%),(\mathrm{b} / \mathrm{c} .3)$ Histogram of real part of unstable modes occurrences $(\%)$.

Table 3: CPU times comparison between Monte Carlo (MC) simulations and both versions of the proposed method: (a) method based on dichotomy and (b) method based on continuation principles; $t_{\text {fit }}$ : time for getting the decomposition, $t_{\text {shoot }}$ : time for evaluating the occurrences.

\begin{tabular}{cccc}
$\begin{array}{c}\text { Number } \\
\text { of points }\end{array}$ & $\begin{array}{c}\text { MC } \\
(\mathrm{s})\end{array}$ & $\begin{array}{c}\text { (a) Dichotomy }(\mathrm{s}) \\
t_{\text {fit }}+t_{\text {shoot }}=t_{\text {total }}\end{array}$ & $\begin{array}{c}\text { (b) Continuation }(\mathrm{s}) \\
t_{\text {fit }}+t_{\text {shoot }}=t_{\text {total }}\end{array}$ \\
\hline 1000 & 345 & $1455+4=1459$ & $788+5=793$ \\
10000 & 3451 & $1455+44=1498$ & $788+46=834$
\end{tabular}


(a)

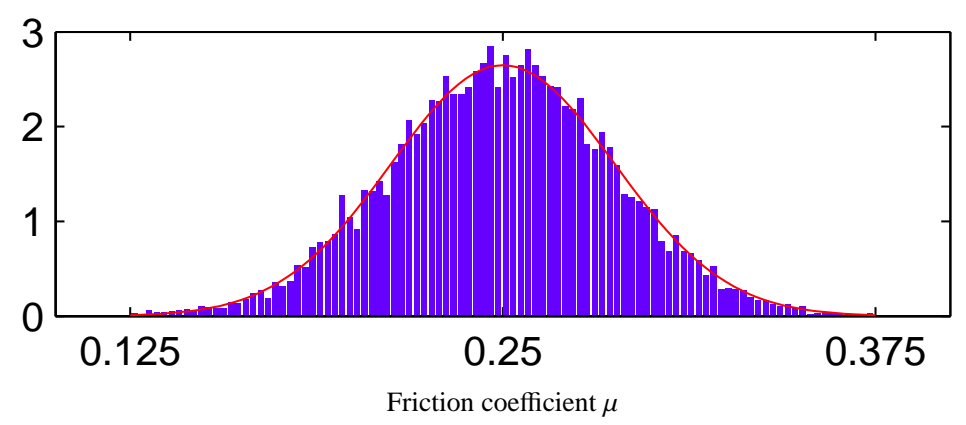

(b.1)

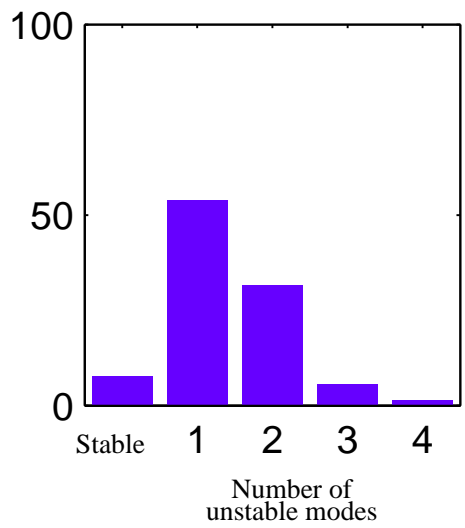

(c.1)

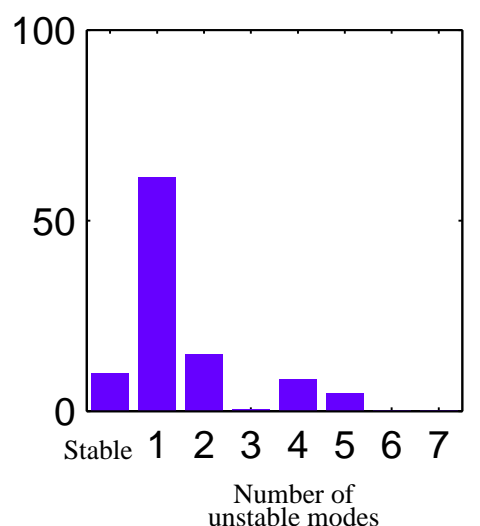

(b. 2 )

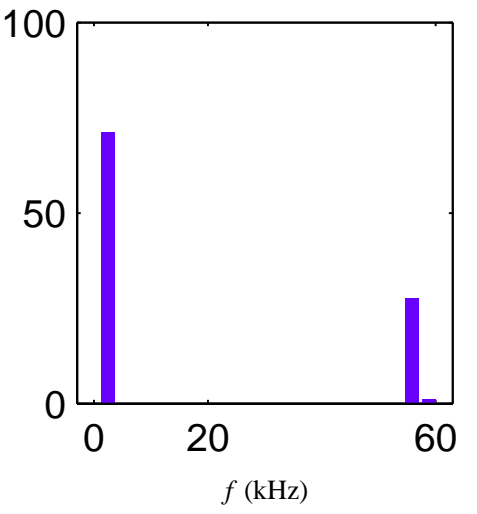

(c.2)

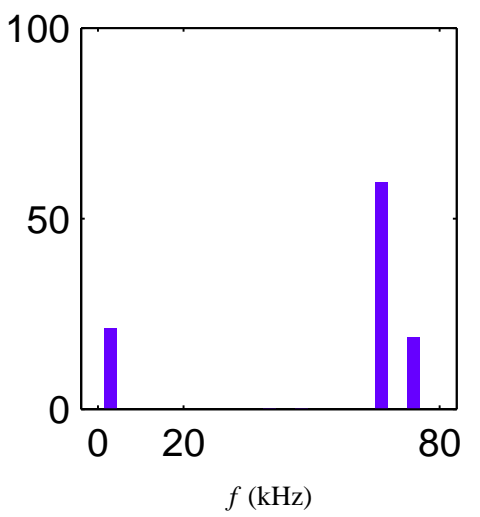

(b.3)

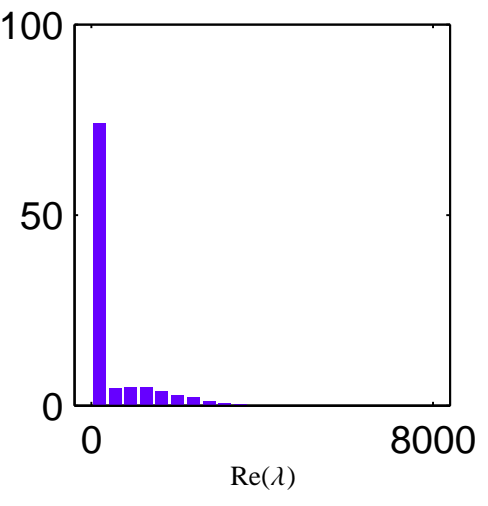

(c.3)

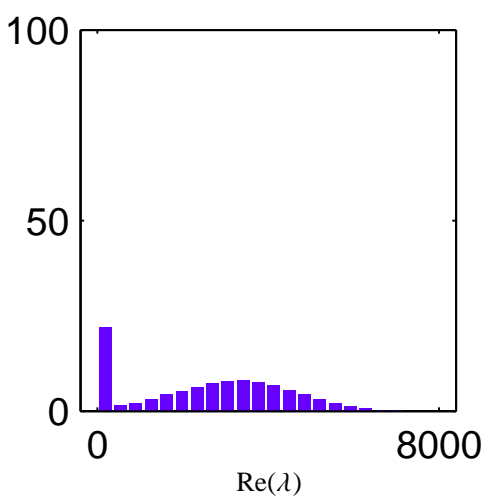

Figure 8: Simulations using a normal law for case 1 ( $\mu$ varies): histograms. (a) Random parameter value occurrences (\%); System stability and squeal information for (b.n) configuration 1 and (c.n) configuration 2; (b/c.1) Histogram of stable/unstable occurrences (\%), (b/c.2) Histogram of unstable frequencies $(\mathrm{kHz})$ occurrences $(\%),(\mathrm{b} / \mathrm{c} .3)$ Histogram of real part of unstable modes occurrences $(\%)$. 
(a.1)

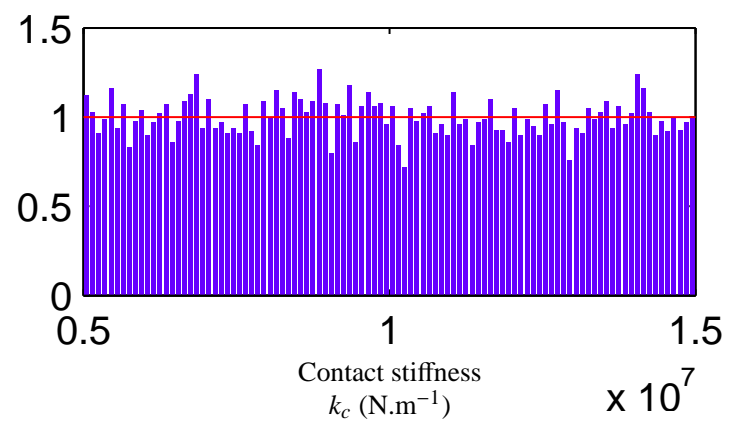

(b.1)

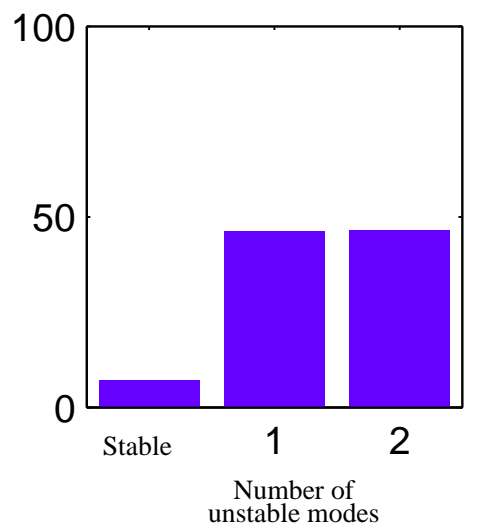

(c.1)

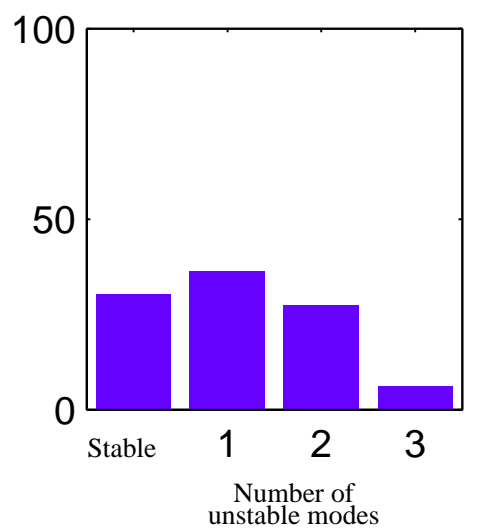

(a.2)

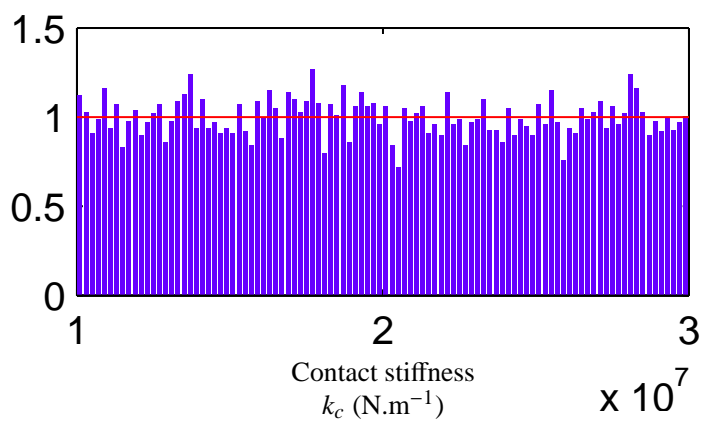

(b.3)

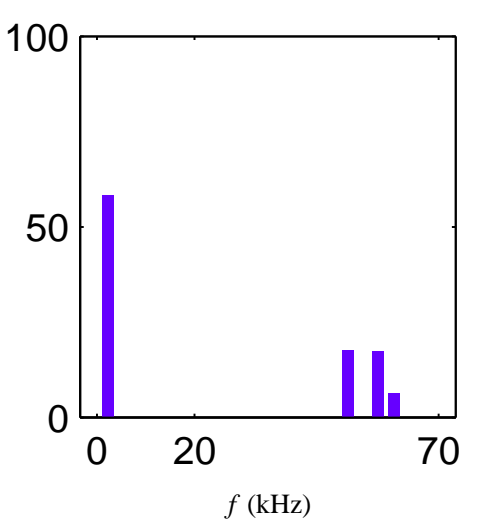

(c. 2)

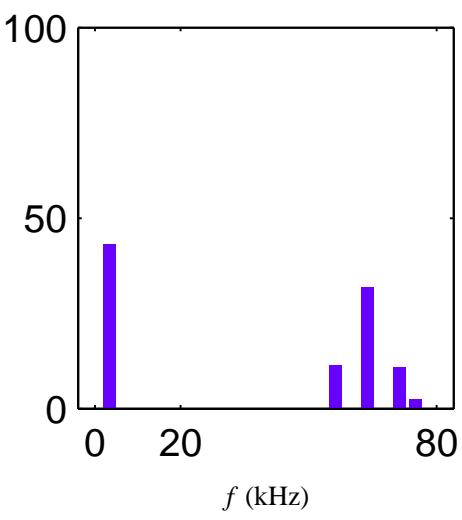

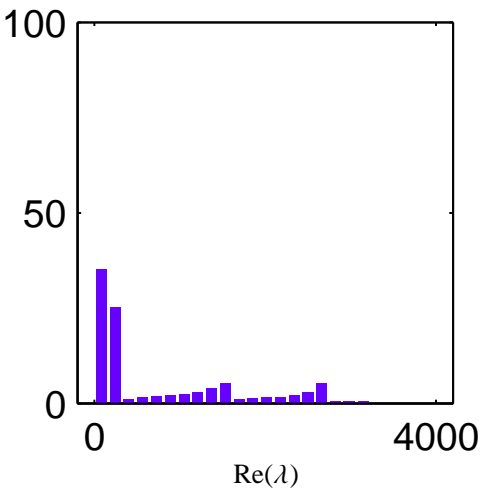

(c.3)

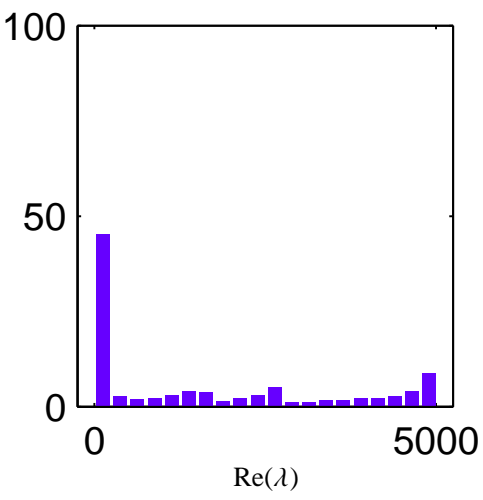

Figure 9: Simulations using a uniform law for case 2 ( $k_{c}$ varies): histograms. (a.x) Random parameter values in (a.1) configuration 1 and (a.2) configuration 2; System stability and squeal information for (b.n) configuration 1 and (c.n) configuration 2; (b/c.1) Histogram of stable/unstable occurrences (\%), (b/c.2) Histogram of unstable frequencies $(\mathrm{kHz})$ occurrences $(\%),(\mathrm{b} / \mathrm{c} .3)$ Histogram of real part of unstable modes occurrences $(\%)$. 
(a.1)

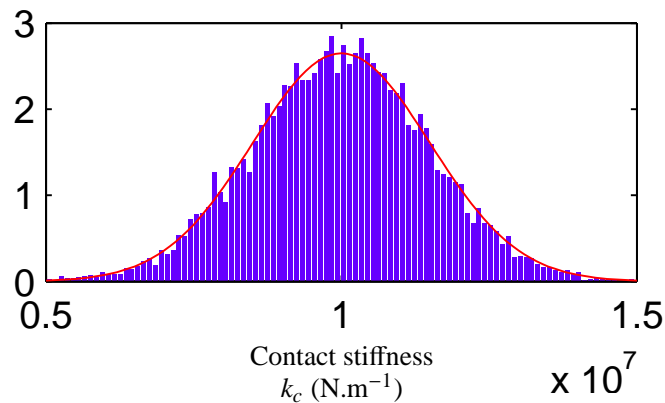

(b.1)

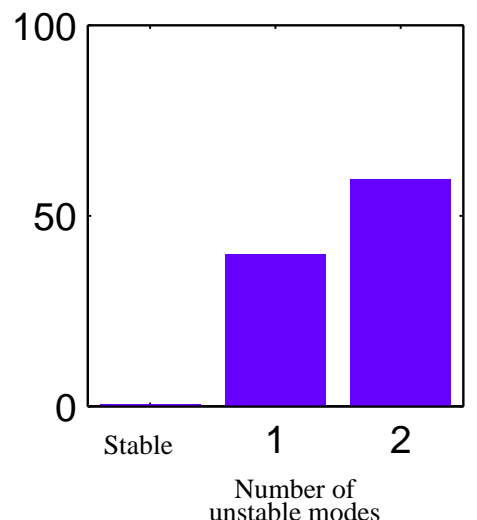

(c.1)

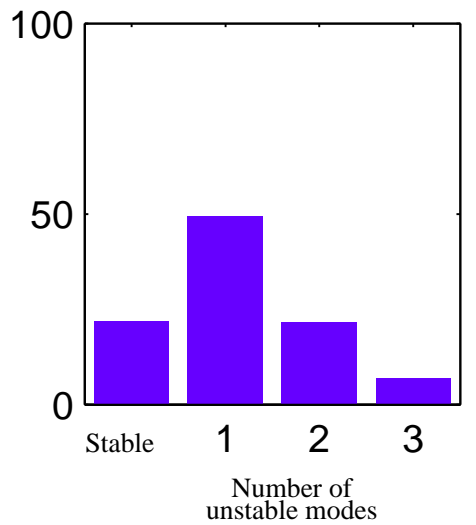

(b. 2$)$

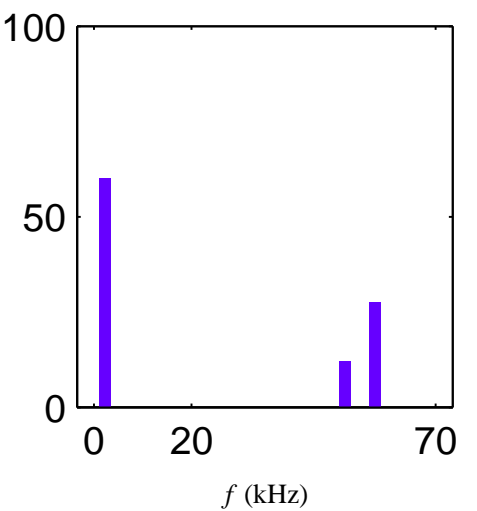

(c.2)

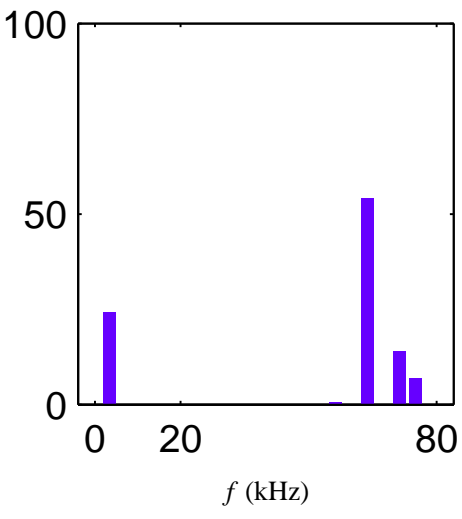

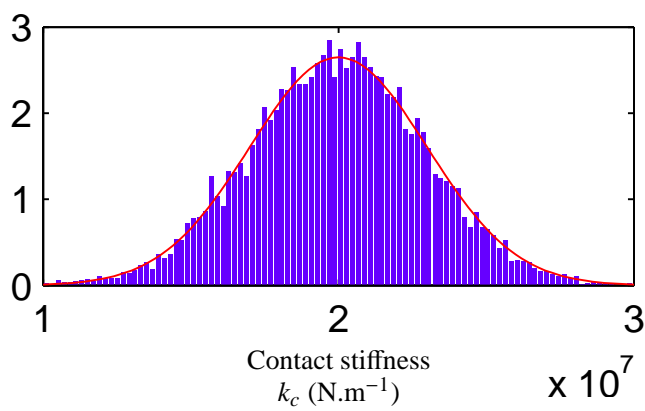

(b.3)

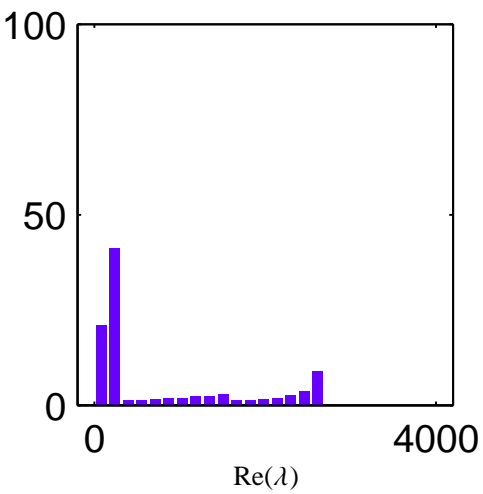

(c.3)

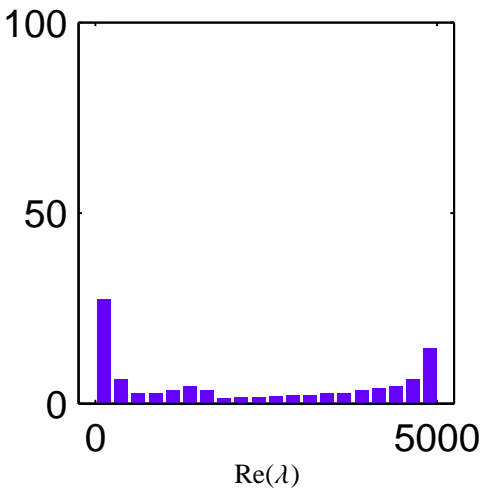

Figure 10: Simulations using a normal law for case 2 ( $k_{c}$ varies): histograms. (a.x) Random parameter values in (a.1) configuration 1 and (a.2) configuration 2; System stability and squeal information for (b.n) configuration 1 and (c.n) configuration 2; (b/c.1) Histogram of stable/unstable occurrences (\%), (b/c.2) Histogram of unstable frequencies $(\mathrm{kHz})$ occurrences $(\%),(\mathrm{b} / \mathrm{c} .3)$ Histogram of real part of unstable modes occurrences $(\%)$. 
or 10000 eigenmodes using their decomposition.

This table shows that the tested method is not advantageous if used on a small sample only (1000 points) for a single input law but may become so if different laws are to be tested. However as soon as a large sample (10000 points) is considered, the method is twice to four times faster than Monte Carlo simulations for a single sample, this ratio increasing with the number of samples or distributions tested. Being also accurate as demonstrated in Section 3.2, this makes it a very interesting method in terms of computational resources consumption.

This table also points out a large advantage for the second version of the method based on continuation principles which is almost twice faster than the first version based on the more simple dichotomy approach.

\section{Conclusions}

This study takes place in the context of uncertain systems. Here, brake squeal noise is studied, viewed as an instability of some modes of the linear system and hence driven by the underlying eigenvalue problem. Uncertain systems can be hard to handle because of the the computation duration in the case of Monte Carlo simulations; hence a class of methods were developed to attempt to avoid the problem of repeated resolutions of a same problem with different values for uncertain parameters. A polynomial basis whose polynomials are functions of the random variables, is used to decompose the eigenmodes - eigenvalues and eigenvectors: this is the Polynomial Chaos, introduced by Ghanem and Spanos in 1991 [25] in the field of structural dynamics. Having the quantities of interest described continuously via polynomials, it is then easy to evaluate them over large samples and hence to compute the statistical estimators such as the mean or higher order moments.

Depending on the problem, one can be confronted with quantities whose variations with respect to the random parameters are not continuous, that is all the more true when studying changes of stability of a dynamic system. Hence, the degree of the polynomial basis must be very large. The size of the system to solve growing with the maximum degree of the chosen basis, one can switch to another strategy and divide the stochastic space, then using a lower degree on each element of the partition: this Multi-Element generalized Polynomial Chaos (MEgPC) method was recently introduced by previous papers, mostly to study equilibria or limit cycles via time integration methods. The present work applied this approach to a structural dynamic system - pad and disc assembly - whose stability is investigated when either the friction coefficient or the contact stiffness is uncertain: the quantities of interest are the eigenmodes, solutions of the eigenvalue problem. The MEgPC method introduced in fluid dynamics is modified in two ways: the classical dichotomy approach to create the partition of the stochastic space is compared to a new version of the method based on continuation principles which happens to be more efficient. Moreover, a criterion to check the decomposition accuracy over the processed cell is proposed. This criterion, developed specifically for the stochastic eigenvalue problem is based on the Rayleigh ratio and lets us evaluate accuracy of the decomposition over a cell without any comparison to the exact values, which makes it an efficient criterion.

Both versions of the method were applied to a reduced finite element model of a brake, consisting of a pad in contact with a disc. Variations of two important yet hard to characterize interface parameters were investigated for two nominal configurations: friction coefficient and contact stiffness; results obtained via the proposed method were compared to Monte Carlo simulations. As the developed method returns a decomposition that does not depend on the random input law, different distributions were tested once the decomposition was obtained. This highlighted both accuracy and efficiency of the method, especially the version implementing continuation principles.

Post-processing of the results are to be examined in two ways.

First, the choice of a random law for description of the varying parameter impacts greatly the stability profile of the brake; as characterizing this random entry is not always easy to perform, the proposed tool presents the advantage to enhance the possibility to test different input laws in a few seconds. Furthermore, the random law used can be whatever is physically thought relevant with no restriction such as being a standard normal law.

Second, comparing the different contact stiffness results, one can conclude that, for the current system, the more stiff the contact is, the less the brake exhibits a squeal propensity but the more unstable modes can coexist outside of stable ranges and the greater the instability growth rate is.

\section{Acknowledgments}

The author gratefully acknowledge the financial support of the French National Research Agency through the Young Researcher program ANR-07-JCJC-0059-01-CSD2.

\section{References}

[1] H. Ouyang, J. E. Mottershead, D. J. Brookfield, S. James, M. P. Cartmell, A methodology for the determination of dynamic instabilities in a car disc brake, International Journal of Vehicle Design 23 (2003) 241-262.

[2] D. Brizard, O. Chiello, J.-J. Sinou, X. Lorang, Performances of some reduced bases for the stability analysis of a disc/pads system in sliding contact, Journal of Sound and Vibration 330 (2011) $703-720$.

[3] N. Coudeyras, J.-J. Sinou, S. Nacivet, A new treatment for predicting the self-excited vibrations of nonlinear systems with frictional interfaces: The constrained harmonic balance method, with application to disc brake squeal, Journal of Sound and Vibration 319 (2009) 1175 - 1199. 
[4] N. Hoffmann, M. Fischer, R. Allgaier, L. Gaul, A minimal model for studying properties of the mode-coupling type instability in friction induced oscillations, Mechanics Research Communications 29 (2002) 197 - 205.

[5] Q. Cao, H. Ouyang, M. I. Friswell, J. E. Mottershead, Linear eigenvalue analysis of the disc-brake squeal problem, International Journal for Numerical Methods in Engineering 61 (2004) 1546-1563.

[6] R. T. Spurr, A theory of brake squeal, in: Proceedings of the Institution of Mechanical Engineers, volume 1, pp. 33-40. 1961.

[7] R. A. Ibrahim, Friction-induced vibration, chatter, squeal, and chaos—part i: Mechanics of contact and friction, Applied Mechanics Reviews 47 (1994) 209-226.

[8] R. A. Ibrahim, Friction-induced vibration, chatter, squeal, and chaos—part ii: Dynamics and modeling, Applied Mechanics Reviews 47 (1994) 227-253.

[9] M. R. North, Disc brake squeal, a theoretical model, Technical Report, Motor Industry Research Association, 1972.

[10] J.-J. Sinou, L. Jézéquel, Mode coupling instability in friction-induced vibrations and its dependency on system parameters including damping, European Journal of Mechanics - A/Solids 26 (2007) 106 - 122.

[11] N. M. Kinkaid, O. M. O’Reilly, P. Papadopoulos, Automotive disc brake squeal, Journal of Sound and Vibration 267 (2003) $105-166$.

[12] H. Ouyang, N. Wayne, Y. Yongbin, F. Chen, Numerical analysis of automotive disc brake squeal: a review, International Journal of Vehicle Noise and Vibration 1 (2005) 207-231.

[13] N. Coudeyras, S. Nacivet, J.-J. Sinou, Periodic and quasi-periodic solutions for multi-instabilities involved in brake squeal, Journal of Sound and Vibration 328 (2009) 520 - 540.

[14] F. Chevillot, J.-J. Sinou, N. Hardouin, L. Jézéquel, Effects of damping on the speed of increase and amplitude of limit cycle for an aircraft braking system subjected to mode-coupling instability, Archive of Applied Mechanics 80 (2010) $1045-1054$.

[15] Y. J. Chern, F. Chen, J. L. Swayze, Nonlinear brake squeal analysis, SAE Papers 2002-01-3138 (2002) $73-76$.

[16] J.-J. Sinou, Transient non-linear dynamic analysis of automotive disc brake squeal - on the need to consider both stability and non-linear analysis, Mechanics Research Communications 37 (2010) 96- 105.

[17] F. Bergman, M. Eriksson, S. Jacobson, Influence of disc topography on generation of brake squeal, Wear 225-229 (1999) $621-628$.

[18] S. Oberst, J. C. S. Lai, Statistical analysis of brake squeal noise, Journal of Sound and Vibration 330 (2011) $2978-2994$.

[19] G. I. Schuëller, H. J. Pradlwarter, Uncertain linear systems in dynamics: Retrospective and recent developments by stochastic approaches, Engineering Structures 31 (2009) 2507 - 2517.

[20] A. Culla, F. Massi, Uncertainty model for contact instability prediction, Journal of the Acoustical Society of America 126 (2009) 1111-1119.

[21] W. K. Liu, T. Belytschko, A. Mani, Random field finite elements, International Journal for Numerical Methods in Engineering 23 (1986) 1831-1845.

[22] T. D. Hien, M. Kleiber, Finite element analysis based on stochastic hamilton variational principle, Computers \& Structures 37 (1990) 893 - 902.

[23] S. Adhikari, Complex modes in stochastic systems, Advances in Vibration Engineering 3 (2004) 1-11.

[24] S. Rahman, Stochastic dynamic systems with complex-valued eigensolutions, International Journal for Numerical Methods in Engineering 71 (2007) 963-986.

[25] R. Ghanem, P. D. Spanos, Stochastic Finite Elements: A Spectral Approach, Springer-Verlag, 1991.

[26] K. Sepahvand, S. Marburg, H. J. Hardtke, Uncertainty quantification in stochastic systems using polynomial chaos expansion, International Journal of Applied Mechanics 2 (2010) 305-353.

[27] C. Soize, A nonparametric model of random uncertainties for reduced matrix models in structural dynamics, Probabilistic Engineering Mechanics 15 (2000) 277 - 294.

[28] R. Murthy, M. P. Mignolet, A. El-Shafei, Nonparametric stochastic modeling of uncertainty in rotordynamics-part i: Formulation, Journal of Engineering for Gas Turbines and Power 132 (2010) 092501. 
[29] R. A. Ibrahim, Structural dynamics with parameter uncertainties, Applied Mechanics Reviews 40 (1987) 309.

[30] C. S. Manohar, R. A. Ibrahim, Progress in structural dynamics with stochastic parameter variations: 1987-1998, Applied Mechanics Reviews 52 (1999) 177-197.

[31] G. I. Schuëller, A state-of-the-art report on computational stochastic mechanics, Probabilistic Engineering Mechanics 12 (1997) $197-321$.

[32] G. I. Schuëller, Computational stochastic mechanics - recent advances, Computers \& Structures 79 (2001) 2225 - 2234.

[33] M. Géradin, D. Rixen, Théorie des Vibrations, Application à la dynamique des structures, Masson, 1993.

[34] D. Xiu, G. E. Karniadakis, The wiener-askey polynomial chaos for stochastic differential equations, SIAM Journal on Scientific Computing 24 (2002) 619-644.

[35] D. Ghosh, R. Ghanem, J. Red-Horse, Analysis of eigenvalues and modal interaction of stochastic systems, AIAA Journal 43 (2005) 2196-2201.

[36] E. Sarrouy, O. Dessombz, J.-J. Sinou, Stochastic analysis of the eigenvalue problem for mechanical systems using polynomial chaos expansion - application to a finite element rotor, Journal of Vibration and Acoustics 134 (2012) 051009-1 - 051009-12.

[37] D. Xiu, G. E. Karniadakis, Modeling uncertainty in flow simulations via generalized polynomial chaos, Journal of Computational Physics 187 (2003) 137 - 167.

[38] N. Wiener, The homogeneous chaos, American Journal of Mathematics 60 (1938) 897-936.

[39] S. Oberst, J. C. S. Lai, Chaos in brake squeal noise, Journal of Sound and Vibration 330 (2011) 955-975.

[40] O. Dessombz, Analyse dynamique de structures comportant des paramètres incertains (Dynamic analysis of structures with uncertain parameters), Ph.D. thesis, Ecole Centrale de Lyon/MEGA, 2000.

[41] X. Wan, G. E. Karniadakis, An adaptive multi-element generalized polynomial chaos method for stochastic differential equations, Journal of Computational Physics 209 (2005) 617 - 642.

[42] J. Le Meitour, D. Lucor, C. J.-C., Prediction of stochastic limit cycle oscillations using an adaptive polynomial chaos method, Journal of Aeroelasticity and Structural Dynamics, Vol 2, No 1 (2010) 2 (2010) 3-22.

[43] E. L. Allgower, K. Georg, Introduction to Numerical Continuation Methods, Springer-Verlag, 2003.

[44] J.-J. Sinou, N. Coudeyras, S. Nacivet, Study of the nonlinear stationary dynamic of single and multi-instabilities for disk brake squeal, International Journal of Vehicle Design 51 (2009) 207-222.

[45] R. J. Allemang, The modal assurance criterion-twenty years of use and abuse, Sound and Vibration 37 (2003) 14-23. 